\title{
On the effect of thermodiffusion on solute segregation during the growth of semiconductor materials by the vertical Bridgman method
}

\author{
Mokhtar Ben Sassi ${ }^{\mathrm{a}}$, Slim Kaddeche ${ }^{\mathrm{a}}$, Marcello Lappa ${ }^{\mathrm{b}}$, Séverine Millet ${ }^{\mathrm{c}}$, Daniel Henry ${ }^{\mathrm{c}}$, Hamda \\ Ben Hadid ${ }^{\mathrm{c}}$ \\ ${ }^{a}$ University of Carthage, Institut National des Sciences Appliquées et de Technologie (INSAT), \\ Laboratoire de Recherche Matériaux, Mesures et Applications, LR 11 ES 25, B.P. 676, \\ 1080 Tunis Cedex, Tunisia \\ ${ }^{b}$ Department of Mechanical and Aerospace Engineering, University of Strathclyde \\ James Weir Building, 75 Montrose Street, Glasgow, G1 1XJ, UK \\ Email: marcello.lappa@strath.ac.uk-marlappa@unina.it
}

${ }^{\mathrm{c}}$ Laboratoire de Mécanique des Fluides et d'Acoustique, CNRS/Université de Lyon, École Centrale de Lyon/Université Lyon 1/INSA de Lyon, ECL, 36 Avenue Guy de Collongue, 69134 Ecully Cedex, France

Email (corresponding author): Slim.Kaddeche@insat.rnu.tn, slimkaddeche@yahoo.fr

\begin{abstract}
The effect of thermodiffusion on dopant distribution in the melt and in the grown crystal is investigated numerically for a vertical Bridgman configuration for situations of pure thermal convection corresponding to dilute alloys. The dopant distribution is shown to be significantly affected by the Soret parameter value. The sensitivity of the system to a variety of parameters, including the Grashof number and the so-called furnace residence time, i.e. the time during which the crucible is maintained in the furnace before initiating the solidification process, is assessed by means of parametric simulations. Moreover, the results indicate that variations in the sign of the Soret parameter can lead to diametrically opposite behaviours, while an increase in the intensity of the thermal convection generally leads to a mitigation of the effects induced by thermodiffusion. On the basis of the numerical results some useful criteria are drawn which could help crystal growers to discern the complex interrelations among the various parameters under one's control (that are not independent of one another) and to elaborate rational guidelines relating to strategies to be used to improve the quality of the resulting crystals.
\end{abstract}

Keywords: A1. Convection; A1. Directional solidification; A1. Segregation; A1. Soret effect; A2. Growth from melt

\section{Introduction}

Crystals of semiconductor materials can be produced by a variety of methods, each displaying its own advantages and bottlenecks [1]. Because the most perfect crystalline arrangement of atoms does not always produce the properties that are needed for specific electronic devices, often small amounts of impurities (dopants) are intentionally added to these materials when they are in the 
liquid state in order to change their ability to conduct electricity (this is the reason why these materials are also often referred to as "alloys").

In general, all these melt growth configurations require the application of thermal gradients across the phase boundary: the axial and/or radial components of such gradients provide driving forces of various natures for free convection in all fluid phases involved. The resulting varying heat-transfer conditions have been a subject of intense research in recent years because they have been found to be directly or indirectly responsible for a vast majority of bulk deficiencies in many materials (indeed, instabilities of the melt flow can lead to oscillatory effects adversely affecting growing crystals, see, e.g., Thevenard et al. [2], Gelfgat et al. [3], Vaux et al. [4] and Lappa [5]). It is also known that in some circumstances, by contrast, enhancing the convective level in the bulk can be instrumental in achieving more homogeneous crystals. As illustrated by Pimputkar and Ostrach [6], Crochet et al. [7], Favier [8] and Müller [9], regardless of the prevailing effect, the resultant fluid motion is always critical to crystal quality.

Research in these fields, therefore, can be seen as a matter of searching for the best conditions promoting optimal crystal growth in terms of product perfection and quality. Along these lines, a variety of methods based on different physical principles and control strategies have been used over the years to influence (damp, mitigate or enhance, depending on the specific situation considered) such flows, e.g. gravity modulation (Bouhallab et al. [10] and Fedoseyev and Alexander [11]), static or rotating magnetic fields (see, e.g., Ben Hadid et al. [12], Kaddeche et al. [13-14], Gelfgat et al. [15] or Dold and Benz [16], respectively), centrifugation (Friedrich et al. [17] and Lan [18]), rotation at fixed rates (Yeckel et al. [19] and Lan [20]) or varying rates (Yeckel and Derby [21] and Wang et al. [22]), thermal modulation (Semma et al. [23-24]) etc. The reader is referred to [25-26] for a more exhaustive review.

Despite all these excellent efforts, however, there are additional important physical aspects, which have been overlooked or not properly taken into account. As an example, although some excellent studies have been appearing where the influence of thermal boundary conditions and thermophysical material properties on velocity configurations in typical Bridgman arrangements (see, e.g., Crochet et al. [7] or Bachran et al. [27]) was properly assessed, the role played by the socalled Soret effect, has not been adequately addressed yet.

Originally discovered by the Swiss scientist Charles Soret in 1879 (although the German Ludwig had described the same phenomenon several years before in a one-page report), this phenomenon, also referred to as thermodiffusion, or the Ludwig-Soret effect (Soret [28-29-30] and Ludwig [31]) is known to induce mass separation in the presence of temperature gradients. Although its exact physical origin is still a matter of debate (see, e.g., Artola and Rousseau [32]), a very interesting interpretation of the mechanism at work can be found, e.g., in Gaeta [33]. Considering the liquid as a seat for elastic waves of all frequencies within certain physical limiting values, which propagate within the liquid mass in all directions, and modelling the flow of heat along the direction of the imposed thermal gradient as an excess flow of undulatory energy in that direction, the thermodiffusion effect was explained on the basis of a "radiation-pressure" model. More precisely, 
by introducing an analogy with some fundamental concepts in the related field of classical mechanics (just as elastic waves can exert dynamic actions on emitting, absorbing, or reflecting surfaces, thermal waves also, being elastic high-frequency acoustic radiation, must exert such a "radiation pressure" along their paths), Gaeta [33] provided a theoretical justification for this effect via the expected mechanical interaction of the aforementioned thermal elastic waves with the matter eventually dispersed in the liquid (small particles or solute molecules). Some years later, Kempers [34-35] elaborated a more comprehensive theory for this effect by incorporating both the thermodynamic contribution from selective attraction/repulsion and the kinetic contribution from selective collision interaction between the components in the considered mixture. Other models have tried to provide a theoretical justification for the change of sign of the Soret coefficient as detailed in Artola and Rousseau [32] and Eslamian [36].

No matter which is the exact mechanism that produces this phenomenon, recently, there has been a resurgence of theoretical studies exploring the consequences that this effect might have in many technological fields. This problem is both fundamentally interesting and important in an array of natural and technological processes. Important examples include chemical and electrochemical reactions, sedimentation equilibrium (e.g., in crude oil reservoirs), transport across membranes, thermosolutal processes in microgravity conditions and cell metabolism (Severinghaus et al. [37], Platten [38], Van Vaerenbergh et al. [39], Mast and Braun [40], Mialdun and Shevtsova [41], Shevtsova et al. [42] ).

For a detailed review of the experimental techniques expressly dedicated to the measurement of thermodiffusion/thermophoresis processes in liquid and gaseous mixtures, the reader may consider Srinivasan and Saghir [43]. Experiments in laboratories on earth as well as in microgravity were discussed in this paper, including a critical review of related diagnostic techniques such as the classical Soret cell, the thermodiffusion column, the two-chamber thermodiffusion cell and the thermal field-flow fractionation, or optical methods such as the classical Soret cell with beam deflection, the thermal diffusion forced Rayleigh scattering, the thermal lens method and the microfluidic fluorescence approach.

Historically, the role potentially played by thermodiffusion during the solidification of binary single- and two-phase systems was originally recognized by Jesse [44]. More than ten years later, Hurle [45] expressly extended the classic Mullins-Sekerka analysis related to the interface morphological stability of crystals growing from dilute binary alloys to include this effect.

Subsequent studies reconsidered these problems from both theoretical and experimental points of view.

As an example, Williams and Philbrook [46] studied liquid Te solutions containing various percentages of $\mathrm{Ag}$. The measurements extended from close to the liquidus temperatures to 1100$1200 \mathrm{~K}$ and were instrumental in determining a correlation between the Soret segregation and the thermoelectric properties of the considered melts. Rudolph et al. [47], examined the case of $\mathrm{Bi}_{1-\mathrm{x}} \mathrm{Sb}_{\mathrm{x}}$ mixed crystals and PbTe, InP, GaAs, CdTe compounds. By means of microgravity experimentation (MFA-067 experiment of the automatic retrievable satellite EURECA), Van Vaerenbergh et al. [48] 
managed to measure the Soret coefficients for the case of tin alloys and compared the resulting data with the predictions of nonequilibrium condensed matter theories. The benefits of microgravity conditions in producing measurements not disturbed by convection effects had been also demonstrated by Praizey [49] during earlier Spacelab missions in 1983 and 1985 for the cases of pure Sn (isotope separation), Sn-Co, Sn-Ag and Sn-Bi.

More recently, with the express intent to identify and address inaccuracies in existing measurements and related theories and propose a better model for the estimation of the thermodiffusion factor, Eslamian et al. [50] derived a general expression for the calculation of such a parameter in binary liquid metal alloys on the basis of a linear non-equilibrium thermodynamics approach similar to that originally developed by Winter and Drickamer [51]. This expression was based on two terms; a first term accounting for the thermally driven interactions between metal ions (a phenomenon similar to that of non-ionic binary mixtures such as hydrocarbons) and a second term, called "electronic contribution", representing the mass diffusion due to the internal electric field that is induced as a result of the imposed thermal gradient. Both terms were cast as functions of the net heats of transport. An interesting methodology was also presented and used to estimate the liquid metal properties, such as the partial molar internal energies, enthalpies, volumes and the activity coefficients used for model validation. The prediction power of the proposed expression along with some other existing thermodiffusion models for liquid mixtures (such as the Haase, Kempers, Drickamer and Firoozabadi formulas) were finally examined against available experimental data obtained on ground or in microgravity environment. These models were refined and compared with other available data by Eslamian and Saghir [52] and Jafar Salehi et al. [53].

Most recently, the relevance of these phenomena to semiconductor processing has been reemphasized by Eslamian and Saghir [54], who have reviewed advances and patents that employ thermodiffusion to fabricate semiconductor, micro-electromechanical systems (MEMS), nanoelectromechanical systems (NEMS), micro and nano thin films.

In the present work, we consider the well-known Vertical Bridgman (VB) technique. Examples of materials treated with this method include pure semiconductors (As, Ag), binaries III-V (GaAs, $\mathrm{GaSb})$ or II-VI (CdTe) systems, ternaries $\left(\mathrm{Ag}_{6} \mathrm{Ge}_{10} \mathrm{P}_{12}, \mathrm{CsMnBr}, \mathrm{Cu}_{12} \mathrm{Sb}_{4} \mathrm{~S}_{13}, \mathrm{Cd}_{1-\mathrm{x}} \mathrm{Zn}_{\mathrm{x}} \mathrm{Te}\right)$, etc. (most of them are doped semiconductors).

Past numerical studies were often concerned with the so-called "inverted" configuration where the system is being heated from below and therefore massive convection takes place (Crespo del Arco et al. [55], Pulicani et al. [56], Larroudé et al. [57], Kaenton et al. [58], Achoubir et al. [59] and Semma et al. [24]). The classical directional solidification (Bridgman) configuration heated from the top, however, is more stable. Most conveniently we concentrate expressly on such a configuration.

In the light of the arguments provided before, control or a proper knowledge of the role played by the Soret effect in these systems should be regarded as an essential topic from a material/product properties optimization perspective. Although a plethora of numerical studies have been appearing over recent years motivated, completely or in part, by a quest to discover means of mitigating 
instabilities and hence of producing single crystals of higher quality for the industry, unfortunately, most of these studies did not consider thermodiffusion and even when they did, this was done under strong limiting assumptions such as purely diffusive conditions (microgravity, Tulcan-Paulescu et al. [60]), one-dimensional modelling (Zheng et al. [61]) or analytic approaches (Alexandrov and Aseev [62]).

To the best of our knowledge only very recently some authors (Jafar-Salehi et al. [63]) applied the finite element method to investigate heat transfer and fluid flow and the effect of thermodiffusion on the vertical solidification of a binary molten metal alloy ( $\mathrm{Sn}-\mathrm{Bi}$ ). Using such approach, the formation of flow field and convection and their effects on temperature distribution, fraction of liquid phase, and concentration distribution were predicted for two cases with and without the thermodiffusion effect. It was shown that the presence of the thermodiffusion modifies the solid phase fraction in the mushy zone, as well as its height in the vertical direction.

A proper consideration of the Soret effect in combination with typical buoyancy flow and related thermosolutal effects in a VB configuration is therefore of a particular importance as the identification of the most dominant phenomenon and/or a proper understanding of the interplay among different mechanisms, may help the researchers in elaborating rational guidelines relating to physical and geometrical factors that can further increase the probability of success in this practical technological process.

Given the stable and axisymmetric nature of the flow typically associated with the classical Bridgman method, our investigations are based on a two-dimensional cylindrical numerical model which solves the Navier-Stokes equations coupled to heat and mass conservation equation in their complete time-dependent and non-linear formulation, completed with the relevant boundary conditions at the solid walls and at the solid/liquid interface. Although the Grashof number is allowed to span several orders of magnitude, we concentrate on the so called "convecto-diffusive" regime (corresponding to typical situations attained in normal gravity conditions when the considered system is subjected to the typical flow control strategies discussed before).

\section{The mathematical model}

\subsection{Directional solidification}

Bridgman growth (named after the American scientist P. W. Bridgman) is based on the well-known concept of directional solidification (Monberg [64]). Obviously, because the whole feed material (above the crystal) is molten (Fig. 1), in VB the presence of an ampoule, which is, for example, a quartz glass or a graphite container, is required for supporting the melt, which may be regarded as the main distinguishing mark with respect to other techniques such as the floating zone method (Lappa [65-67]).

As molten material lies above solid material, the system is generally characterized by a stable thermal stratification which should guarantee no convection in the theoretical case of a perfectly 
planar (horizontal) crystal/melt interface. Due to unavoidable departure from ideal conditions (see, e.g. Lan [20]), however, radial temperature gradients develop, which, in turn, lead to the onset of flows of gravitational nature, typically located in proximity to the solidification (crystal/melt) front. Such a front, which is deformed owing to its interplay with convective and thermal effects for dilute alloys, in turn, plays a significant role in determining the dynamics of crystallization and structural organization of the dopant into the solid.

In practice, this topic must be placed on a more precise theoretical context by introducing some necessary concepts and notions. Such a theoretical mixture is propaedeutic to a better recognition, definition and characterization of the aforementioned phenomena. From a theoretical standpoint, in general, the (dimensional) boundary condition for the dopant at the melt/crystal interface in the absence of the Soret effect can be written as:

$$
\left.\left(D_{C} \underline{\hat{n}} \cdot \underline{\nabla} C\right)\right|_{z=S_{c r y}}+\left.(1-k) \frac{\partial S_{c r y}}{\partial t}\left(\underline{\hat{n}} \cdot \underline{i}_{z}\right) C\right|_{z=S_{c y y}}=0
$$

where $\mathrm{C}$ is the dopant concentration (more precisely the dopant mass fraction), $\mathrm{k}$ is the segregation coefficient (an intrinsic property of the considered material), representing the ratio between the dopant concentration in the crystal $\left(\mathrm{C}_{\text {cry }}\right)$ and the dopant concentration in the melt at the interface $\left(\mathrm{C}\left(\mathrm{z}=\mathrm{S}_{\mathrm{cry}}\right)\right), \mathrm{D}_{\mathrm{c}}$ is the diffusion coefficient of the dopant in the melt, $\mathrm{S}_{\text {cry }}=\mathrm{S}_{\text {cry }}(\mathrm{r}, \mathrm{t})$ is the function expressing the dependence of the interface shape on the radial coordinate (and time), $\underline{\underline{i}}_{z}$ and $\underline{\hat{n}}$ are unit vectors along the vertical $z$ axis and the direction locally normal to the interface, respectively (Fig. 1). Note that the Stefan flow, which could be important at an interface with a gas phase, is generally negligible at a melt/crystal interface and has been ignored in the study.

\subsection{Soret coefficient and related quantities}

In order to properly account for the Soret effect, Eq. (1) has to be reformulated as:

$$
\left.\left(D_{C} \underline{\hat{n}} \cdot \underline{\nabla} C\right)\right|_{z=S_{c r y}}+\left.\left(D_{T} C(1-C) \underline{\hat{n}} \cdot \underline{\nabla} T\right)\right|_{z=S_{c r y}}+\left.(1-k) \frac{\partial S_{c r y}}{\partial t}\left(\underline{\hat{n}} \cdot \underline{i}_{z}\right) C\right|_{z=S_{c r y}}=0
$$

where the second term describes the Soret effect, with $D_{T}$ the thermodiffusion coefficient. This new boundary condition reflects the well-known expression introduced by Platten [38] for the mass flux of a dopant in a dilute binary mixture:

$$
J_{c}=-\rho D_{C} \underline{\nabla} C-\rho D_{T} C(1-C) \underline{\nabla T}
$$

where $\rho$ is the density of the mixture.

\subsection{Considered configuration}

We consider a two-dimensional cylindrical model of the vertical Bridgman growth (Fig. 1). The solidified interface is assumed to move at a constant pre-determined rate $\left(V_{f}\right)$, which is the displacement velocity of the crucible with respect to the constant temperature gradient imposed by 
the furnace. $V_{f}$ is considered as small, so that the solidification front is stable and its shape is not far from a parabola with a moderate deflection. This deflection is fixed and taken to be $10 \%$ of the radius crucible in this work.

\subsection{Governing equations}

The vertical Bridgman with the Soret effect should be regarded as a rather complex system, and we cannot hope to fully explain its dynamics as the analytic solution to a small set of equations. Rather, a full understanding requires describing it in a consistent way on many levels simultaneously. One of these levels involves simulating the flow by numerically solving the governing equations of motion as completely as possible. The main motivation of the present section is the selection of characteristic scales for length, time, velocity, temperature and so on, so that the governing equations are conveniently made dimensionless. We use the following reference quantities for length, time and velocity:

$L_{\text {ref }}=R$

(the new dimensionless space variables are denoted as $r^{*}$ and $z^{*}$, and the dimensionless length associated with $S_{c r y}$ is denoted as $S$ ),

$t_{r e f}=\frac{R^{2}}{v}$

$v_{\text {ref }}=\frac{v}{R}$

where $\mathrm{R}$ is the system radius and $v$ is the fluid kinematic viscosity. The nondimensional temperature is introduced as:

$\theta=\frac{T-T_{c}}{R \nabla T}$

where $T_{\mathrm{c}}$ is the temperature along the growth interface. Finally, the dimensionless concentration is defined as:

$c=\frac{C}{C_{0}}$

where $C_{0}$ is the initial uniform concentration of dopant species in the melt.

Because we consider the limit of a very dilute alloy, the temperature along the growth interface $T_{\mathrm{c}}$, the buoyancy term in the momentum equation, and the Soret term are all assumed to be independent of the dopant concentration $\mathrm{C}$. The dependence of density on temperature is taken into account via the well-known Boussinesq approximation. The coefficient in the Soret term is taken at $C_{0}$ and expressed as $D_{T} C_{0}\left(1-C_{0}\right)$.

The domain containing the melt, which varies during the solidification process, is transformed into a fixed, normalized domain using the following transformation: $z=z^{*} / S(r, t)$ and $r=r^{*}$ (see, e.g., Lappa et al. [65] for additional details on the application of variables-change techniques to problems with deformed interfaces). For convenience, the notation $r$ and $z$ is chosen for these new normalized space variables which vary between 0 and 1 . 
In the framework of a vorticity-stream-function formulation, the nondimensional momentum equation in the melt can be cast in condensed form in this normalized domain as:

$$
\begin{aligned}
& \frac{\partial \varsigma}{\partial t}+u \frac{\partial \varsigma}{\partial r}+\left(v-z v_{F}\right) \frac{1}{S} \frac{\partial \varsigma}{\partial z}+\frac{\varsigma u}{r}=\frac{\partial^{2} \varsigma}{\partial r^{2}}+\frac{1}{r} \frac{\partial \varsigma}{\partial r}+\frac{1}{S^{2}} \frac{\partial^{2} \varsigma}{\partial z^{2}}-\frac{\varsigma}{r^{2}}-G r \frac{1}{S} \frac{\partial \theta}{\partial r} \\
& \frac{\partial^{2} \psi}{\partial r^{2}}-\frac{1}{r} \frac{\partial \psi}{\partial r}+\frac{1}{S^{2}} \frac{\partial^{2} \psi}{\partial z^{2}}=r \varsigma
\end{aligned}
$$

The energy and dopant species conservation equations in the melt are:

$$
\begin{aligned}
& \frac{\partial \theta}{\partial t}+u \frac{\partial \theta}{\partial r}+\left(v-z v_{F}\right) \frac{1}{S} \frac{\partial \theta}{\partial z}=\frac{1}{P r}\left(\frac{\partial^{2} \theta}{\partial r^{2}}+\frac{1}{r} \frac{\partial \theta}{\partial r}+\frac{1}{S^{2}} \frac{\partial^{2} \theta}{\partial z^{2}}\right) \\
& \frac{\partial c}{\partial t}+u \frac{\partial c}{\partial r}+\left(v-z v_{F}\right) \frac{1}{S} \frac{\partial c}{\partial z}=\frac{1}{S c}\left(\frac{\partial^{2} c}{\partial r^{2}}+\frac{1}{r} \frac{\partial c}{\partial r}+\frac{1}{S^{2}} \frac{\partial^{2} c}{\partial z^{2}}+\sigma\left(\frac{\partial^{2} \theta}{\partial r^{2}}+\frac{1}{r} \frac{\partial \theta}{\partial r}+\frac{1}{S^{2}} \frac{\partial^{2} \theta}{\partial z^{2}}\right)\right)
\end{aligned}
$$

where

$$
\begin{aligned}
& u=\frac{1}{r} \frac{1}{S} \frac{\partial \psi}{\partial z} \\
& v=-\frac{1}{r} \frac{\partial \psi}{\partial r} \\
& \varsigma=\frac{1}{S} \frac{\partial u}{\partial z}-\frac{\partial v}{\partial r}
\end{aligned}
$$

$S$ is the dimensionless height of the liquid zone by which the height is normalized and it is defined as:

$S(r, t)=\frac{S_{c r y}(r, t)}{R}=A-v_{F} t-\Delta \xi r^{2}$

where $A=\frac{H}{R}$ is the aspect ratio of the crucible ( $H$ is the height of the crucible), $\Delta \xi R$ is the interface deflection taken equal to $0.1 \mathrm{R}\left(10 \%\right.$ of the crucible radius) and $v_{F}=\frac{R V_{F}}{v}$ is the dimensionless interface velocity.

Different nondimensional parameters appear in the equations (6)-(9). $\sigma=S_{T} C_{0}\left(1-C_{0}\right) \frac{\mathrm{R} \nabla T}{C_{0}}=S_{T}\left(1-C_{0}\right) \mathrm{R} \nabla T$ is referred to as the Soret parameter $\left(S_{T}=\frac{D_{T}}{D_{c}}\right.$ being known as the Soret coefficient, see Platten [38]). Note that $\sigma$ positive (negative) means that, under the action of the Soret effect, the dopant migrates toward the cold (hot) part of the melt. The other relevant characteristic numbers are the Prandtl number, $\operatorname{Pr}=\frac{v}{\alpha}$, the Schmidt number, $S c=\frac{v}{D_{c}}$, the Grashof number, $G r=\frac{g \beta_{T} \nabla T R^{4}}{v^{2}}$, and the Peclet number, $P e=\frac{R V_{F}}{D_{c}}=v_{F} S c$, where $\alpha$ is the melt thermal diffusivity, $\beta_{T}$ is the thermal expansion coefficient, and $\nabla T$ is the constant rate of temperature increase imposed by the heating facility along the axial direction $z$. 


\subsection{Boundary conditions}

For problem closure, the momentum, energy, and concentration equations are complemented with the relevant boundary conditions, which can be cast in compact form as:

$u=v=0, \frac{\partial c}{\partial z}+\sigma \frac{\partial \theta}{\partial z}=0$ and $\theta=S(r=1, t)$ at $z=0$,

$u=v=0, \frac{1}{S}\left(\frac{\partial c}{\partial z}+\sigma \frac{\partial \theta}{\partial z}\right)=P e(1-k) c-2 r \Delta \xi\left(\frac{\partial c}{\partial r}+\sigma \frac{\partial \theta}{\partial r}\right)$ and $\theta=0$ at $z=1$ (growth interface),

$u=\frac{\partial v}{\partial r}=0, \frac{\partial c}{\partial r}+\sigma \frac{\partial \theta}{\partial r}=0$ and $\frac{\partial \theta}{\partial r}=0$ at $r=0$

$u=v=0, \frac{\partial c}{\partial r}+\sigma \frac{\partial \theta}{\partial r}=0$ and $\theta=S(r=1, t)(1-z)$ at $r=1$.

The condition for the temperature at $r=1$ is a linear profile corresponding to the temperature gradient imposed by the heating facility (constant temperature gradient furnace). The concentration in the solid is finally determined via its relationship with the concentration at the interface on the liquid side, i.e. $c_{S}=k c_{L}$.

\subsection{Numerical method and validation}

The governing equations (6)-(9) supplemented with the related boundary conditions (Eqs. (12) and (13)) are solved using a stream-function-vorticity $(\psi-\zeta)$ method. The major advantage of this approach relies on the possibility of solving the equations leaving aside problems related to the pressure gradient (the presence of this term is removed through application of the curl operator to the momentum equation, which leads to Eq. (6)) (see Lappa [25]). The resulting finite-difference method has been implemented here via an ADI (alternating direction implicit) technique with forward differences for time derivatives and Hermitian relationships for spatial derivatives with a truncation error of $\mathrm{O}\left(\Delta \mathrm{t}^{2}, \Delta \mathrm{r}^{4}, \Delta \mathrm{z}^{4}\right)$ (Roux et al. [68]).

The mesh used to solve the problem has been generated on the basis of the Thompson technique. In such a context, an a priori grid tailoring study for structured grids has been carried out towards the end of quantifying the improvement of resolution achievable by grid adaptation. On the basis of such a mesh refinement study, in particular, we have decided to use the same $25 \mathrm{x} 101$ grid that already proved to give sufficiently accurate results in the earlier work by Kaddeche et al. [69]. Such a mesh (see Fig. 2) is not uniform. Grid density is larger in proximity to the sidewalls, especially in proximity to the growth interface where the most interesting phenomena are expected to take place (in terms of thermal convection and solute rejection).

Our algorithm was validated through comparison with available results in the literature (Coriell and Sekerka [70] and Motakef [71]) for different conditions and convection levels. The reader is referred to Kaddeche et al. [69] and Ben Hadid et al. [12] for additional details on the numerical method and the code validation process. 


\section{Results}

The problem with five distinct degrees of freedom defined in Sect. 2 is too complex and broad in scope. In the absence of observational information to properly constrain the model, a reduction of the parameters is beneficial. For the sake of concreteness, we consider a melt with $\operatorname{Pr}=0.015, \mathrm{Sc}=10$ and $k=0.087$ and corresponding to an effective Ge-Ga alloy. In order to get a realistic order of magnitude for the values to be used for $\sigma$, we refer to the work of Tulcan-Paulescu et al. [60] where a Ge-Ga alloy was considered as in the present study (the values of $\sigma$ derived from their work can range up to 1). With regard to the other relevant parameters, we assume that the normalized interface deflection is $\Delta \xi=10 \%$, the dimensionless interface velocity is $v_{\mathrm{F}}=-0.2$ and the initial aspect ratio $A$ is taken equal to 4 . Specific numerical examples are conceived and presented to provide inputs for an increased understanding of the underlying fluid flow mechanisms and the interplay with the Soret effect. In particular, most of the results are presented at a time $t$ corresponding to the situation (relevant and interesting from an engineering point of view) in which $50 \%$ of the melt present at the initial time $t=0$ has undergone solidification (solidified fraction equal to 0.5$)$. Note finally that these results are given in the (physical) space spanned by the $\left(r^{*}, z^{*}\right)$ coordinates.

\subsection{Effect of the Soret parameter at $G r=510^{3}$}

We first consider a case in the so-called convecto-diffusive regime (corresponding to moderate values of the Grashof number) and the influence of the Soret effect will be studied for both positive and negative values of the Soret parameter.

The dopant distribution in the melt and in the crystal when half the sample has been solidified is shown in Fig. 3 for $\mathrm{Gr}=510^{3}$ and different values of $\sigma$. As $\mathrm{Gr}$ is fixed these different cases correspond exactly to the same flow, which is up along the axis of the sample and returns along the lateral boundaries. Indeed, the flow induced in these melts considered as dilute alloys is only due to thermal convection (the solutal contribution to convection is negligible) and is thus not affected by the thermodiffusion effect. Moreover, this thermal convection is driven by the temperature field, which is almost diffusive for the low-Pr-value melts considered $(\operatorname{Pr}=0.015)$.

The numerical results shown in Figs. 3a-3b for $\sigma=0$ and $\sigma=1$ reveal that for positive values of $\sigma$ the isoconcentration lines retain essentially the same morphology as for $\sigma=0$, but they are more numerous as they cover a larger concentration range. Indeed, a stronger enrichment in dopant in the crystal can be noticed for $\sigma>0$ with respect to the reference situation in which $\sigma=0$, connected to a stronger enrichment of the melt close to the growth interface. The discrepancy between these concentration levels has been found to become more significant with an increase in the Soret parameter $\sigma$, which clearly indicates the important role played by the Soret effect. In fact, as already seen, the Soret effect modifies the mass-flux (Eq. (3)), with a contribution connected with the temperature gradient, and this will affect the species balance equation (Eq. (9)), as well as the zero- 
mass-flux boundary conditions. For positive values of the Soret parameter, according to Eq. (3), the dopant will migrate under the influence of the Soret effect towards the cold part of the sample, i.e. towards the growth interface. It will then enhance the dopant concentration in this zone, which was already the rich zone due to the rejection at the interface (the maximum value is increased from 4.67 to 5.89). A consequence is the enrichment of the crystal with a maximum dopant concentration increasing from 0.41 to 0.54 . In contrast, the Soret effect will be responsible for the depletion of solute in the relatively hot area close to the crucible top wall, making this zone still poorer in dopant (the minimum value is decreased from 1.20 to 0.36 ). Note that, due to the presence of an almost constant temperature gradient in the main parts of the melt, the Soret term $\sigma \Delta \theta$ (where $\Delta$ is the Laplacian operator) in the concentration equation remains weak. This means that the species moved by the Soret effect will not accumulate in the core, but rather migrate to the boundaries, where they are collected and diffuse back to the core.

The influence of the Soret effect for negative values of $\sigma$ is shown in Fig. 3c for $\sigma=-1$. In this case, the Soret effect affects more clearly the isoconcentration lines and the global concentration pattern. In fact, for negative values of the Soret parameter, the dopant migration due to the Soret effect is opposite to what occurs for $\sigma \geq 0$ : the dopant migrates towards the hot zone of the melt, at the top of the crucible, enriching this originally poor zone and leading to the depletion of the interface zone (the maximum value of dopant concentration near the interface decreases from 4.67 for $\sigma=0$ to 3.24 for $\sigma=1$ ). Due to the enrichment of the upper zone, the minimum dopant concentration (with a value equal to 1.66) is no more in this zone, but in the descending flow zone near the lateral boundaries. In contrast, the dopant depletion in the neighborhood of the solid-liquid interface leads to a poorer grown crystal with a maximum dopant concentration decreased to 0.28 .

Additional information on the variation of the dopant concentration during these different solidification cases can be obtained by following the time evolution of the "axial" and "radial" segregations. They are defined at each time step (i.e. at each position in $z^{*}$ of the interface, from $S=A=4$ to $S=\mathrm{A} / 2=2)$ as

$$
C s_{a v}=\frac{\int_{0}^{1} r C_{s}(r, S) d r}{\int_{0}^{1} r d r}
$$

and

$$
\Delta C=\frac{C_{s}(r, S)_{\max }-C_{s}(r, S)_{\min }}{C s_{a v}},
$$

respectively. In these expressions, $C_{s}(r, S)$ is the concentration along the growth interface in the crystal $\left(C_{s}(r, S)=k C(r, S)\right.$ where $k=0.087$ and $C(r, S)$ is the concentration along the growth interface in the liquid), and $C_{s}(r, S)_{\max }\left(C_{s}(r, S)_{\min }\right)$ is its maximum (minimum) value along the interface. These characteristic variables are used as indicators of the overall dopant concentration level and concentration homogeneity along the crystal, respectively. They will be given as a function of the dimensionless solidified length $l_{s}\left(l_{s}=A-S\right)$, which varies from 0 to 2 . 
The evolution of these quantities along the crystal for the same value $\mathrm{Gr}=510^{3}$ and different values of the Soret parameter $\sigma$ is shown in Fig. 4. Concerning the axial segregation (Fig. 4a), which naturally increases along the crystal due to the dopant rejection at the interface, it is strengthened by positive values of the Soret parameter $\sigma$ and weakened by negative values. For example, for $l_{s}=2$, the increase of the axial segregation when $\sigma$ is changed from 0 to 1 is about $28 \%$, and a quite similar value is found for the decrease obtained when $\sigma$ is changed from 0 to -1 . The corresponding radial segregation is shown in Fig. 4b, characterized by a strong initial increase leading to a kind of plateau with only weakly varying values. The influence of the Soret effect on this radial segregation is similar to what was found for the axial segregation: a positive value of the Soret parameter $\sigma$ will increase the radial segregation, whereas a negative value will decrease it. The stronger influence found for the negative values of $\sigma$ is mainly connected to the fact that $\Delta C$ is normalized by the axial segregation, which is smaller for these values of $\sigma$. To sum up, when $\sigma$ is positive (negative), the grown crystal becomes richer (poorer) in dopant species and less (more) homogeneous.

\subsection{Convective effects: influence of the Grashof number}

Earlier studies on the impact of the Soret effect on directional solidification (in particular, on the distribution of the dopant in both solid and liquid phases) were essentially concerned with the idealized conditions provided by a microgravity environment [60] and investigated by one dimensional models that provide no information on the radial segregation and consequently the crystal homogeneity. We want to focus here on more realistic situations representative of typical ground (terrestrial) conditions and analyze numerically the influence of the convection level on the segregation in presence of the Soret effect with a two dimensional axisymmetric model. For that, the Grashof number Gr used in our numerical computations will span the overall range from $\mathrm{Gr}=0$ (corresponding to a purely diffusive regime, i.e. what is obtained in microgravity conditions) up to $\mathrm{Gr}=10^{6}$ (corresponding to the so-called mixed regime), with intermediate values representative of the convecto-diffusive regime.

The dopant distribution in the melt and in the crystal when half the sample has been solidified is shown for different values of $\sigma$ in Figs. 5-7 for $\mathrm{Gr}=0,2.510^{4}$ and $10^{6}$, respectively. If we refer to the maximum concentration in the crystal (or in the melt), the results indicate that the higher the convection level is, the smaller the differences among the different cases $\sigma=0.5, \sigma=-0.5$ and $\sigma=0$ are. Indeed, the higher levels of convection obtained when $\mathrm{Gr}$ is increased will homogenize the melt, decreasing the segregation due to the dopant rejection at the interface, but also that induced by the Soret effect. The concentration patterns change with $\mathrm{Gr}$, with a mixing at the scale of the melt for $\mathrm{Gr}=2.510^{4}$ and a mixing more concentrated in the lower half-part of the melt (closer to the interface) for $\mathrm{Gr}=10^{6}$. Note that for the negative value of $\sigma$, the minimum concentration in the melt is still moved from the upper boundary: this effect is particularly enhanced for the intermediate value of Gr. 
The axial and radial segregations are also plotted for four values of $\mathrm{Gr}\left(\mathrm{Gr}=0,2500,510^{5}\right.$ and $\left.10^{6}\right)$ and three values of $\sigma(\sigma=0,0.5$ and -0.5$)$ in Fig. 8. The discrepancy due to thermodiffusion, which affects the profiles of axial segregation (Fig. 8a), is clearly reduced when increasing the Grashof number from the small values $(\mathrm{Gr}=0$ and 2500$)$ to the large values $\left(\mathrm{Gr}=510^{5}\right.$ and $\left.10^{6}\right)$. The maximum discrepancy between the values of $C s_{a v}$ for $\sigma=0$ and $\sigma=0.5$ evolves from 0.0714 for $\mathrm{Gr}=0$ to 0.0225 for $\mathrm{Gr}=10^{6}$. Concerning the radial segregation (Fig. 8b), the situation is somewhat different as the difference between the cases $\sigma=0$ and $|\sigma|=0.5$ rather undergoes an increase with Gr. In particular, the maximum discrepancy between the values of $\Delta C$ for $\sigma=0$ and $\sigma=0.5$ evolves from 0.0156 for $\mathrm{Gr}=0$ to 0.0418 for $\mathrm{Gr}=10^{6}$.

The results discussed in this section have first shown that the Soret effect can have a major influence in weak flow cases with regard to the axial segregation. This can concern all the situations in which a control of the convection by decreasing its intensity is being attempted, e.g. via the use of magnetic fields. The situations with a mushy zone, which develops in the vicinity of the growth interface and where the convective flow is damped, could also be affected in a privileged way by the Soret effect. At the opposite, the influence of Soret effect is enhanced for the well mixed regime cases with regard to radial segregation. Consequently, the effect of thermodiffusion should not be neglected whatever the convective level, for a reliable prediction of macrosegregation in crystals grown by this technique.

\subsection{Effect of the furnace residence time before the start of the solidification process}

For all the simulations discussed in the preceding sections, the solidification process was assumed to start without any delay and, consequently, the dopant concentration in the melt at the beginning of the growth process was assumed to be constant, i.e. $C=C_{0}=1$ (homogeneous repartition of the dopant species in the whole molten phase). In this scenario, the initial values of axial and radial segregation are ideally equal to $C s_{a v}=k C_{0}=k=0.087$ and $\Delta C=0$, respectively.

More realistic scenarios, however, can be considered, in which the crucible is maintained in the furnace for a certain time before initiating the solidification process (this time will be referred to as $t_{b}$ in the following). During this time, the thermodiffusion will be acting to modify the concentration distribution in the molten phase, so that the simulations will have to consider this modified non-uniform concentration distribution as the initial stage of the solidification evolution. This initial concentration distribution will indeed be non-uniform along the solid-liquid growth interface, giving initial values $C s_{a v} \neq 0.087$ and $\Delta \mathrm{C} \neq 0$.

To illustrate these effects, the variation of $C s_{a v}$ and $\Delta C$ for $\mathrm{Gr}=5000$ and different values of $t_{b}$ for $\sigma=0.5$ and $\sigma=-0.5$ have been plotted in Fig. 9. As expected, these plots show initial values of $C s_{a v}$ and $\Delta C$ which have been affected by thermodiffusion in the pre-solidification stage. More precisely, the initial values of $C s_{a v}$ increase with $t_{b}$ for $\sigma>0$ and decrease with $t_{b}$ for $\sigma<0$ (Fig. 9a). Concerning $\Delta C$ which is, by definition, a positive quantity, an increase with $t_{b}$ of its initial value can be noticed regardless of the specific value of $\sigma$ considered (Fig. 9b). Interestingly, 
however, this increase is effective on the whole range of $t_{b}$ values ( $t_{b}$ between 0 and 40 ) for $\sigma=-0.5$, whereas an almost constant value is reached as soon as $t_{b}=4$ for $\sigma=0.5$ (despite the fact that $C s_{a v}$ increases on the whole range of $t_{b}$ ).

Considering now the solidification stage, it can be observed that the modifications generated by thermodiffusion on $\mathrm{Cs}_{a v}$ in the pre-solidification stage induce similar modifications during the whole solidification process, i.e. an increase of $t_{b}$ leads to an enrichment of the crystal for $\sigma=0.5$, whereas it produces a depletion for $\sigma=-0.5$ (Fig. 9a). These variations in the level of concentration of the dopant in the crystal are particularly significant in the early stages of the solidification process and tend to become less perceptible when the solidified length approaches $l_{s}=2$. In fact, just after the beginning of the solidification process, the increasing rate of solute rejected in the melt along the growth interface, namely $P e(1-\mathrm{k}) \mathrm{c}(\mathrm{Eq} .12 \mathrm{~b})$, becomes dominant compared to the almost constant contribution resulting from thermodiffusion (almost unchanged quasi-diffusive temperature field due to the low Prandtl number of the melt, i.e., $\operatorname{Pr}=0.015$ ). Such a situation leads to the loss of sensitivity of the system to the initial conditions during the final stages of the directional solidification process.

Additional information is given by the behavior of the radial segregation in Fig. 9b. This quantity is slightly affected by $t_{b}$ for $\sigma=0.5$ as only a weak increase of $\Delta C$ can be seen for $0 \leq t_{b} \leq 40$. The influence on $\Delta C$, however, which was imperceptible for $4 \leq t_{b} \leq 40$ in the pre-solidification stage, becomes visible and is then maintained during the whole solidification process. For $\sigma=-0.5$, the results are significantly different. During the early stages of the solidification process, the radial segregation created by thermodiffusion during the pre-solidification stage is strongly reduced by the rejection of dopant along the solid-liquid interface. The further advancement of the interface will allow the radial segregation to rise again in proximity to the growth interface, but the influence of $t_{b}$ on $\Delta C$ is now inverted, i.e. the radial segregation decreases with increasing $t_{b}$. As for $C s_{a v}$, this influence is strong when the solidified length is about $l_{s}=0.5$, but becomes less perceptible at the end of the solidification process for $l_{s}=2$.

The most remarkable outcome of these findings comes from the possibility for the crystal growers to use the trends disclosed by the numerical investigation to optimize the overall growth process. For instance, the level of axial segregation can be monitored by adjusting the time during which the crucible is maintained in the furnace before beginning the solidification process, allowing to fulfill compositional requirements imposed by different applications.

\section{Conclusion}

Although extensive studies on the vertical Bridgman configuration are reported in the literature, the majority of this work has been aimed primarily at either, (i) the assessment of the influence exerted on the overall process by thermophysical material properties, (ii) the investigation of fluid-dynamic instabilities, (iii) the process improvement to enable such materials to be produced successfully on a large scale (via the application of proper stabilization techniques). The effort so far provided on 
such a subject has yielded many positive results, but also many unanswered questions. This field continues to burgeon and bring surprises to this day.

Let us recall that obtaining a robust understanding requires a diversity of model types, ranging from simple to complex, in which various processes are turned on and off and the results carefully diagnosed; this is called a modeling hierarchy and its use forms the backbone of forward progress in any field of technology. Along these lines, this work has been devoted to clarify the role played in such cases by the so-called Soret effect, which has attracted so much attention over recent years in many companion technological fields.

Recent application-driven technological demands require vastly superior control of crystal of doped semiconductor materials grown by the Bridgman technique with respect to the current state-of-theart. Thermodiffusion phenomena are inherent to exerting such control. Towards this end, an "ad hoc" configuration and a related mathematical model have been defined with the specific intent to separate the effects produced by thermodiffusion from those eventually emerging as a consequence of complex three-dimensional and oscillatory flow instabilities.

Along these lines, numerical computations, based on a two-dimensional axisymmetric transient modelling of the vertical Bridgman directional solidification process, have been performed to expressly investigate the role of thermodiffusion on the distribution of dopant species in both the molten and solidified phases (liquid and crystal).

Our results show that thermodiffusion can significantly affect the composition of the grown crystal depending on the intensity of buoyancy convection occurring in the liquid phase. When the convection level is relatively low the axial segregation is significantly affected by thermodiffusion. At the opposite, when the well mixed regime is attained (larger values of the Grashof number), the effects of thermodiffusion tend to be less significant on the axial segregation but have an increasing impact on radial segregation and consequently on the crystal homogeneity.

The value of the Soret parameter (especially its sign) of course also plays a role in such dynamics. Indeed, for positive values of $\sigma$, the grown crystal becomes richer in dopant species, although the concentration is, in general, less uniform. In contrast, for negative values of $\sigma$, the grown ingot is poorer, but more homogeneous in terms of concentration of the dopant species.

We also explored the sensitivity of the system to the so-called furnace residence time, i.e. the time during which the crucible is maintained in the furnace before initiating the solidification process, revealing some interesting dynamics.

Finally, it is perhaps worth pointing out that convection damping effects, which are favorable to thermodiffusion impact, should also be expected (even without using an external control such as a magnetic field) in the so-called mushy zone which develops in the vicinity of the growth interface. This was the subject of the very recent analysis by other authors (Jafar-Salehi et al. [63]), which should be regarded as the natural theoretical counterpart of the present work. An improved understanding of all these aspects is likely to yield important economic benefits, for example better process control, increased efficiency, improved reproducibility and the capacity to modify or tailor products for specific applications. 


\section{References}

[1] Handbook of Crystal Growth (Second Edition), Bulk Crystal Growth, A volume in Handbook of Crystal Growth, Edited by Peter Rudolph (2015) Elsevier.

[2] Thevenard D., Rouzaud A., Comera J., Favier J.J., "Influence of convective thermal oscillations on a solidification interface in Bridgman growth", J. Cryst. Growth 108 (1991) 572-582.

[3] Gelfgat A.Yu., Bar-Yoseph P.Z., Solan A., "Axisymmetry breaking instabilities of natural convection in a vertical Bridgman growth configuration", J. Cryst. Growth 220 (2000) 316-325.

[4] Vaux S., BenHadid H., Henry D., "Study of the hydrodynamic instabilities in a differentially heated horizontal circular cylinder corresponding to a Bridgman growth configuration", J. Cryst. Growth 290 (2006) 674-682.

[5] Lappa M., "Secondary and oscillatory gravitational instabilities in canonical three-dimensional models of crystal growth from the melt. Part 2: Lateral heating and the Hadley circulation", C. R. Mecanique 335 (2007) 261-268.

[6] Pimputkar S.M., Ostrach S., "Convective effects in crystals grown from melt", J. Cryst. Growth 55 (1981) 614-646.

[7] Crochet M.J., Dupret F., Ryckmans Y., Geyling F.T., Monberg E.M., "Numerical simulation of crystal growth in a vertical Bridgman furnace", J. Cryst. Growth 97 (1989) 173-185.

[8] Favier J.J., "Recent advances in Bridgman growth modelling and fluid flow", J. Cryst. Growth 99 (1990) $18-29$.

[9] Müller G., "Convective instabilities in melt growth configurations", J. Cryst. Growth 128 (1993) 26-36.

[10] Bouhallab S., Henry G., Boschetti E., Naumann R.J, "Stabilizing/destabilizing effects of axial accelerations in Bridgman growth", J. Cryst. Growth 165 (1996) 129-136.

[11] Fedoseyev A.I., Alexander J.I.D., "Investigation of vibrational control of convective flows in Bridgman melt growth configurations", J. Cryst. Growth 211 (2000) 34-42.

[12] Ben Hadid H., Henry D., Kaddeche S., "Numerical study of convection in the horizontal Bridgman configuration under the action of a constant magnetic field. Part I. Two-dimensional flow", J. Fluid Mech. 333 (1997) 23-56.

[13] Kaddeche S., Henry D., Putelat T., Ben Hadid H., "Instabilities in liquid metals controlled by constant magnetic field. Part I: Vertical magnetic field", J. Cryst. Growth 242 (2002) 491-500.

[14] Kaddeche S., Henry D., Putelat T., Ben Hadid H., "Instabilities in liquid metals controlled by constant magnetic field. Part II: Horizontal magnetic field", J. Cryst. Growth 242 (2002) 501-510.

[15] Gelfgat A.Yu., Bar-Yoseph P.Z., Solan A., "Effect of axial magnetic field on three-dimensional instability of natural convection in a vertical Bridgman growth configuration", J. Cryst. Growth 230 (2001) 63-72.

[16] Dold P., Benz K. W., "Modification of fluid flow and heat transport in vertical Bridgman configurations by rotating magnetic fields", Cryst. Res. Tech. 32 (1997) 51-60.

[17] Friedrich J., Baumgartl J., Leister H.-J., Mueller G., "Experimental and theoretical analysis of convection and segregation in vertical Bridgman growth under high gravity on a centrifuge", J. Cryst. Growth 167 (1996) 45-63.

[18] Lan C.W., "Effects of centrifugal acceleration on the flows and segregation in vertical Bridgman crystal growth with steady ampoule rotation", J. Cryst. Growth 229 (2001) 595-600.

[19] Yeckel A., Doty F. P., Derby J. J., "Effect of steady crucible rotation on segregation in high-pressure vertical Bridgman growth of cadmium zinc telluride", J. Cryst. Growth 203 (1999) 87-102.

[20] Lan C.W., "Effects of ampoule rotation on flows and dopant segregation in vertical Bridgman crystal growth", J. Cryst. Growth 197 (1999) 983-991.

[21] Yeckel A., Derby J.J., "Effect of accelerated crucible rotation on melt composition in high-pressure vertical Bridgman growth of cadmium zinc telluride", J. Cryst. Growth 209 (2000) 734-750.

[22] Wang L.C., Liu Y.C., Yu W.C., Roux B., Lyubimova T.P., Lan C.W., "Segregation control of vertical Bridgman growth of Ga-doped germanium crystals by accelerated crucible rotation: ACRT versus angular vibration", J. Cryst. Growth 311 (2009) 684-687.

[23] Semma E., El Ganaoui M., Timchenko V., Leonardi E., "Unsteadiness and control by using thermal boundary modulation in restricted fluid domain of vertical Bridgman apparatus", Int. J. Materials and Product Technology 22 (2005) 122-134. 
[24] Semma E.A., El Ganaoui M., Timchenko V. and Leonardi E., "Thermal modulation effects on thermosolutal convection in a vertical Bridgman cavity", Fluid Dyn. Mater. Process 6 (2010) 233-250.

[25] M. Lappa, "Thermal convection: Patterns, evolution and stability", John Wiley \& Sons, Ltd (2009), Chichester, England).

[26] M. Lappa, "Rotating thermal flows in natural and industrial processes", John Wiley \& Sons, Ltd (2012), Chichester, England).

[27] Bachran A., Reinshaus P., Seifert W., "Influence of thermal processing parameters and material properties on velocity configurations in semiconductor melts during the vertical Bridgman growth technique", Cryst. Res. Technol. 33 (1998) 27-36.

[28] Soret Ch., "Sur l'état d'équilibre que prend au point de vue de sa concentration une dissolution saline primitivement homogène dont deux parties sont portées à des températures différentes", Arch. Sci. Phys. Nat. 2 (1879) 48-61.

[29] Soret Ch., "Influence de la température sur la distribution des sels dans leurs solutions, C. R. Acad. Sci. 91 (1880) 289-291.

[30] Soret Ch., "Sur l'état d'équilibre que prend au point de vue de sa concentration une dissolution saline primitivement homogène dont deux parties sont portées à des températures différentes", Ann. Chim. Phys. 22 (1881) 293-297.

[31] Ludwig C., "Diffusion zwischen ungleich erwwärmten orten gleich zusammengestzter lösungen", Sitz. Ber. Akad. Wiss. Wien Math-Naturw. K1. 20 (1856) 539.

[32] Artola P.A., Rousseau B., "Microscopic interpretation of a pure chemical contribution to the Soret effect", Phys. Rev. Lett. 98 (2007) 125901.

[33] F. S. Gaeta, "Radiation pressure theory of thermal diffusion in liquids", Phys. Rev. 182 (1969) 289-296.

[34] Kempers L.J.T.M., "A thermodynamic theory of the Soret effect in a multicomponent liquid", J. Chem. Phys. 90 (1989) 6541.

[35] Kempers L.J.T.M., "A comprehensive thermodynamic theory of the Soret effect in a multicomponent gas, liquid, or solid", J. Chem. Phys. 115 (2001) 6330.

[36] Eslamian M., "Advances in thermodiffusion and thermophoresis (Soret Effect) in liquid mixtures", Front. Heat Mass Transf. 2 (2011) 043001.

[37] Severinghaus J.P., Sowers T., Brook E.J., Alley R., Bender M., "Timing of abrupt climate change at the end of the Younger Dryas interval from thermally fractionated gases in polar ice", Nature 391(1998) 141-146. [38] Platten J.K., "The Soret effect: A review of recent experimental results", J. Appl. Mech. 73 (2006) 5-15.

[39] Van Vaerenbergh S. R., Saghir Z., Daridon J.L., Luhmer M., Galand Q., Galliéro G., Montel F., Bickert J., Legros J.C., "Experimental microgravity Soret coefficients in multicomponent systems: towards mixing rules?", High Temperatures-High Pressures 39 (2010) 53-56.

[40] Mast C.B., Braun D., "Thermal trap for DNA replication", Phys. Rev. Lett. 104 (2010) 188102.

[41] Mialdun A., Shevtsova V., "Measurement of the Soret and diffusion coefficients for benchmark binary mixtures by means of digital interferometry", J. Chem. Phys. 134 (2011) 044524.

[42] Shevtsova V., Mialdun A., Melnikov D., Ryzhkov I., Gaponenko Y., Saghir Z., Lyubimova T., Legros J.C., "The IVIDIL experiment onboard the ISS: Thermodiffusion in the presence of controlled vibrations", C.

R. Mecanique 339 (2011) 310-317.

[43] Srinivasan S., Saghir M. Z., "Experimental approaches to study thermodiffusion - A review", Int. J. Therm. Sci. 50 (2011) 1125-1137.

[44] Jesse R.E., "The Soret effect and the solidification of binary single- and two-phase systems", J. Cryst. Growth 5 (1969) 132-134.

[45] Hurle D.T.J., "The effect of Soret diffusion on the morphological stability of a binary alloy crystal", J. Cryst. Growth 61 (1983) 463-472.

[46] Williams R. K., Philbrook W. O., "The Soret effect in molten Ag-Te solutions", J. Electrochem. Soc. 128 (1981) 1034-1040.

[47] Rudolph P., Boeck T., Schmidt P., "Thermodiffusion and morphological stability in convectionless crystal growth systems from melts and melt-solutions", Cryst. Res. Tech. 31 (1996) 221-229.

[48] Van Vaerenbergh S., Garandet J.P., Praizey J.P., Legros J.C., "Reference Soret coefficients of natural isotopes and diluted alloys of Tin", Phys. Rev. E 58 (1988) 1866-1873.

[49] Praizey J.P., "Benefits of microgravity for measuring thermotransport coefficients in liquid metallic alloys", Int. J. Heat Mass Transfer 32 (1989) 2385-2401. 
[50] Eslamian M., Sabzi F., Saghir M.Z., "Modeling of thermodiffusion in liquid metal alloys", Phys. Chem. Chem. Phys. 12 (2010) 13835-13848.

[51] Winter F.R., Drickamer H.G., "Thermal diffusion in liquid metals", J. Phys. Chem. 59 (1955) 12291230 .

[52] Eslamian M., Saghir M.Z., "Thermodiffusion (thermomigration) and convection in molten semiconductor-metal layers", Int. J. Therm. Sci. 50 (2011) 1232-1242.

[53] Jafar-Salehi E., Eslamian M., Saghir M.Z., "Estimation of molecular and thermodiffusion coefficients for non-ideal molten metal alloys and its implication in solidification process", Can. J. Chem. Eng. 92 (2014) 1314-1324.

[54] Eslamian M. and Saghir M. Z., (2012), Thermodiffusion Applications in MEMS, NEMS and Solar Cell Fabrication by Thermal Metal Doping of Semiconductors, Fluid Dynamics \& Materials Processing, 8(4), 353-380.

[55] Crespo del Arco E., Pulicani J.P., Bontoux P., "Simulations and analysis of time-dependent convection in low Pr fluids", Int. J. Phys. Chem. Hydrodyn. 11 (1989) 681-692.

[56] Pulicani J.P., Crespo Del Arco E., Randriamampianina A., Bontoux P., Peyret R., "Spectral simulation of oscillatory convection at low Pr", Int. J. Num. Meth. Fluids 10 (1990) 481-517.

[57] Larroudé P., Ouazzani J., Alexander J.I.D., Bontoux P., "Symmetry breaking transition and oscillatory flows in a 2D directional solidification model", Eur. J. Mech, B/Fluids 13 (1994) 353-381.

[58] Kaenton J., Semma A., Timchenko V., El Ganaoui M., Leonardi E., de Vahl Davis G., "Effects of anisotropy and solid/liquid thermal conductivity ratio on flow instabilities during inverted Bridgman growth", Int. J. Heat Mass Transfer 47 (2004) 3403-3413.

[59] Achoubir K., Bennacer R., Cheddadi A., El Ganaoui M., Semma E., "Numerical study of thermosolutal convection in enclosures used for directional solidification (Bridgman cavity)", Fluid Dyn. Mater. Process. 4 (2008) 199-210.

[60] Tulcan-Paulescu E., Balint A.M., Balint S., "The effect of the initial dopant distribution in the melt on the axial compositional uniformity of a thin doped crystal grown in strictly zero-gravity environment by Bridgman-Stockbarger method", J. Cryst. Growth 247 (2003) 313-319.

[61] Zheng L.L., Larson D.J. Jr., Zhang H., "Role of thermotransport (Soret effect) in macrosegregation during eutectic/off-eutectic directional solidification", J. Cryst. Growth 191 (1998) 243-251.

[62] Alexandrov D.V., Aseev D.L., "One-dimensional solidification of an alloy with a mushy zone: thermodiffusion and temperature-dependent diffusivity", J. Fluid Mech. 527 (2005) 57-66.

[63] Jafar-Salehi E., Eslamian M., Saghir M.Z., "Effect of thermodiffusion on the fluid flow, heat transfer, and solidification of molten metal alloys", Engineering Science and Technology, an International Journal 19 (2016) 511-517.

[64] Monberg E., "Bridgman and related growth techniques". In Handbook of Crystal Growth (ed. D.T.J. Hurle) 2 (1994) 53-97. North-Holland, Amsterdam.

[65] Lappa M., Yasushiro S., Imaishi N., "3D numerical simulation of on ground Marangoni flow instabilities in liquid bridges of low Prandtl number fluid", Int. J. Num. Meth. Heat Fluid Flow 13 (2003) 309-340.

[66] Lappa M., "Combined effect of volume and gravity on the three-dimensional flow instability in noncylindrical floating zones heated by an equatorial ring", Phys. Fluids 16 (2004) 331-343.

[67] Lappa M., "Analysis of flow instabilities in convex and concave floating zones heated by an equatorial ring under microgravity conditions", Comput. Fluids 34 (2005) 743-770.

[68] Roux B., Bontoux P., Loc T.P., Daube O., in: Lecture Notes in Mathematics, Vol. 771, Ed. R. Rautmann (Springer, Berlin, 1980) pp. 450-468.

[69] Kaddeche S., Garandet J.P., Barat C., Ben Hadid H., Henry D., "Interface curvature and convection related macrosegregation in the vertical Bridgman configuration", J. Cryst Growth. 158 (1996) 144-152.

[70] Coriell S.R., Sekerka R.F., "Lateral solute segregation during unidirectional solidification of a binary alloy with a curved solid-liquid interface", J. Cryst. Growth 46 (1979) 479-482.

[71] Motakef S., "Interference of buoyancy-induced convection with segregation during directional solidification: Scaling laws", J. Cryst. Growth 102 (1990) 197-213. 


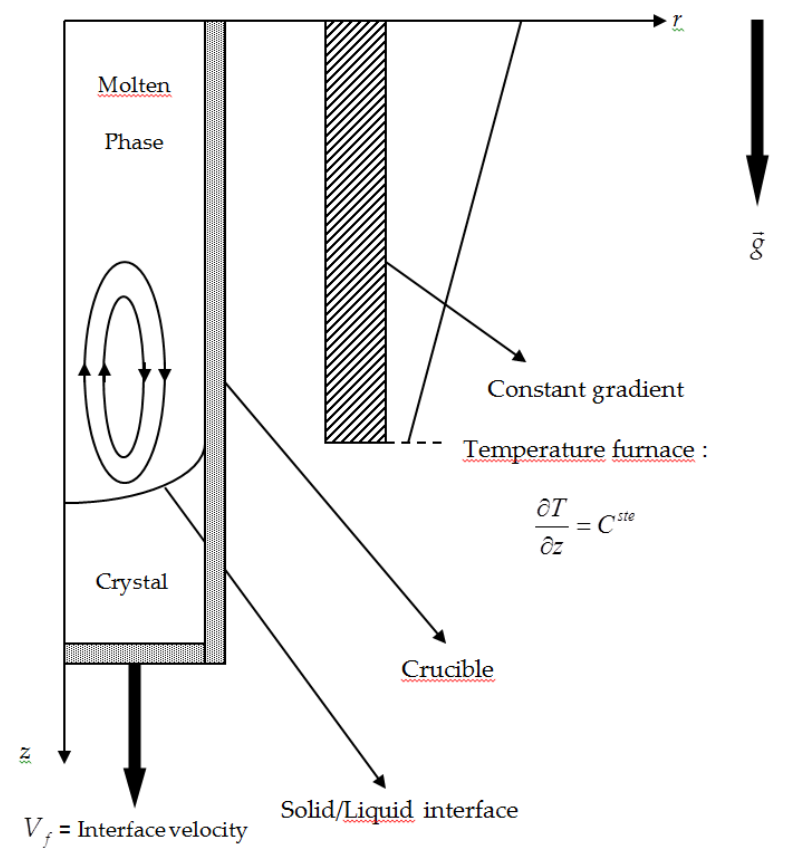

Fig. 1. Sketch of Vertical Bridgman (VB) technique.

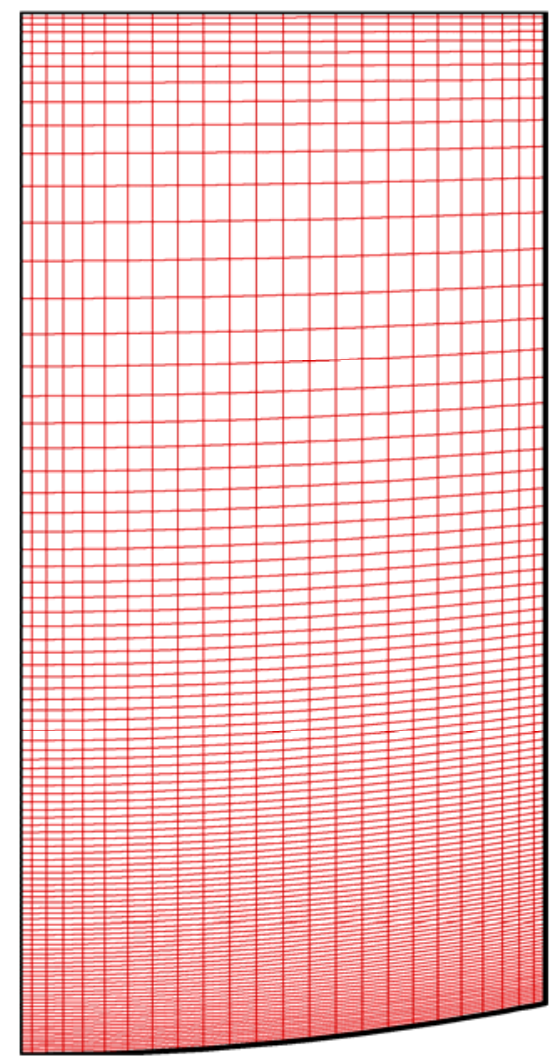

Fig. 2. Nonuniform mesh (with $(25,101)$ grid points) used for the numerical simulations and presented in the $r^{*}, z^{*}$ coordinates at mid-solidification. 


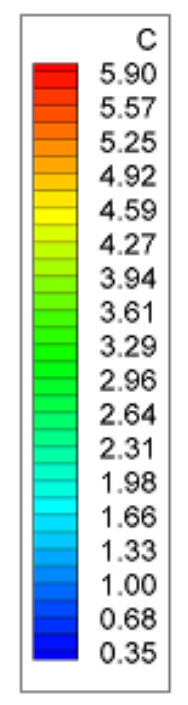

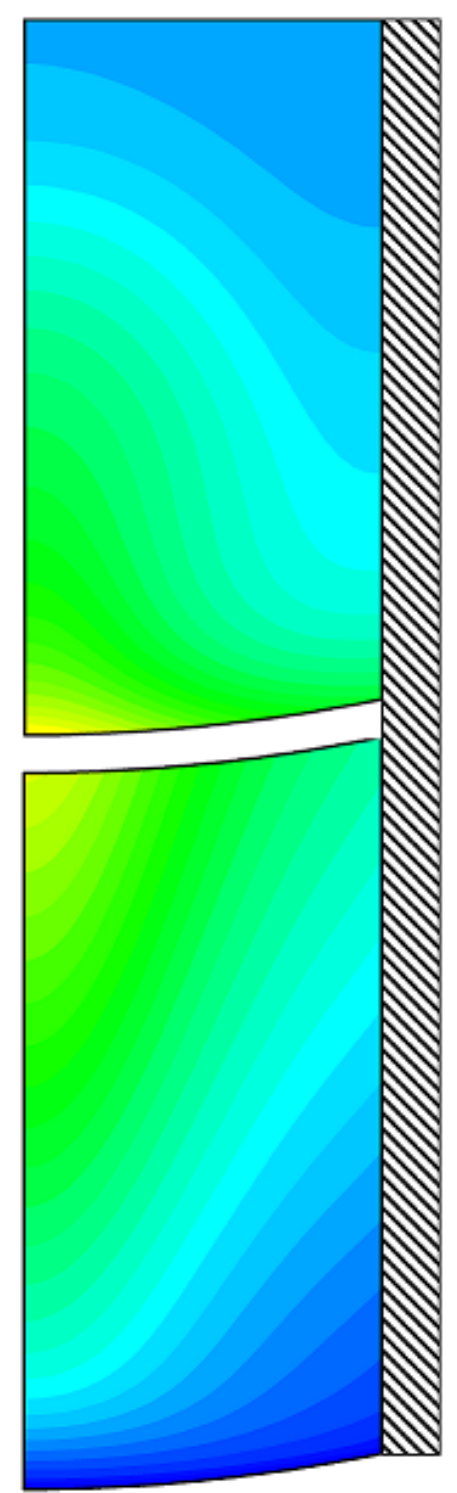

(a)

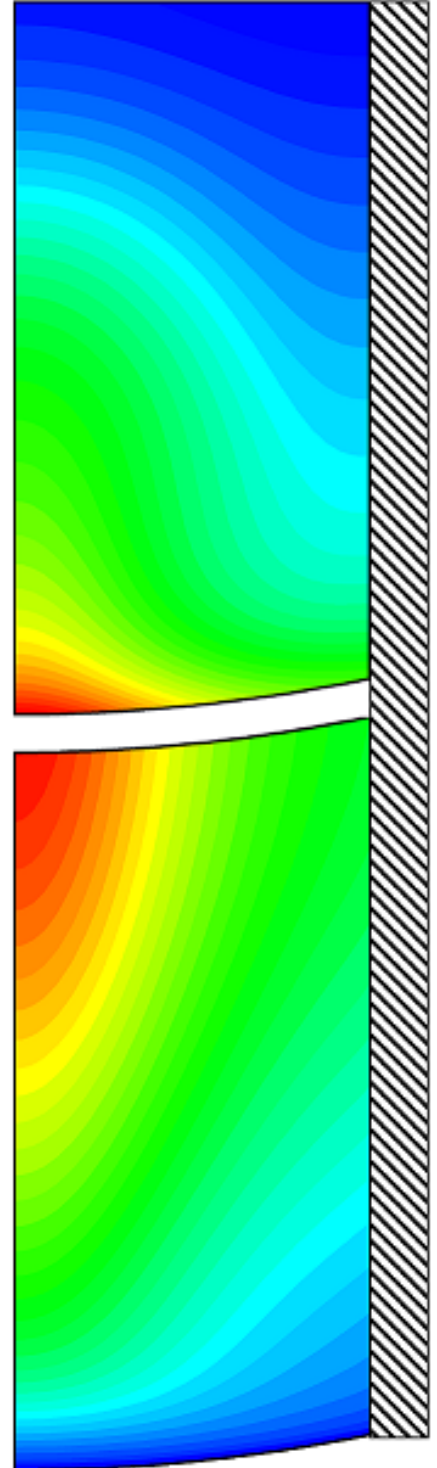

(b)

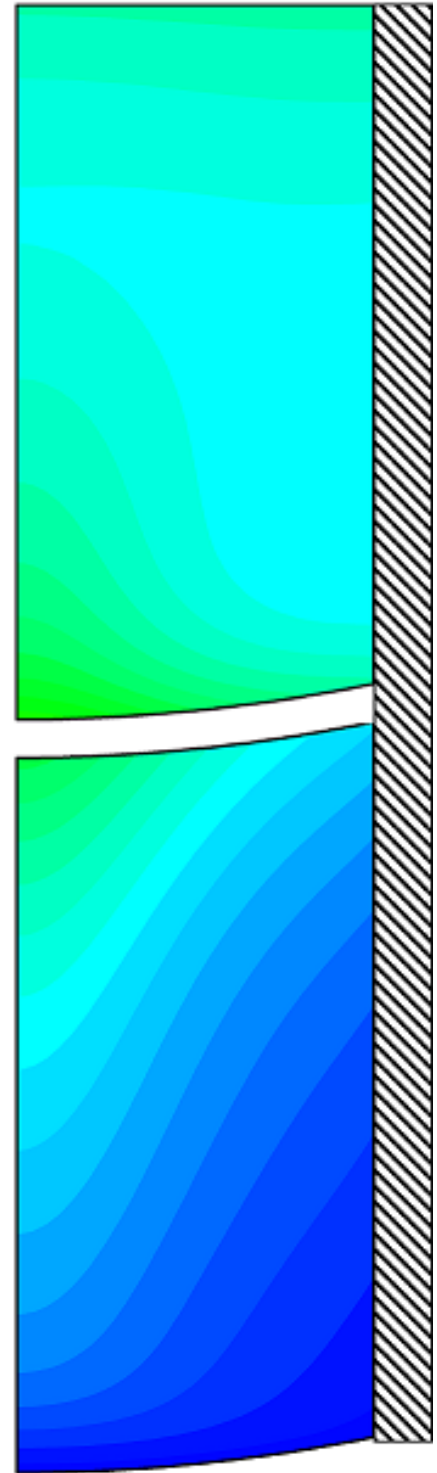

(c)

Fig. 3. Dopant distribution in the melt (top views) and in the crystal (bottom views) when half the sample has been solidified for $\mathrm{Gr}=5000, \sigma=0$ (a), $\sigma=1$ (b) and $\sigma=-1$ (c). The minimum and maximum dopant concentrations in the melt are 1.20 and 4.67 (a), 0.36 and 5.89 (b), 1.66 and 3.24 (c). The maximum dopant concentration in the solid is 0.41 (a), 0.53 (b), 0.28 (c). 

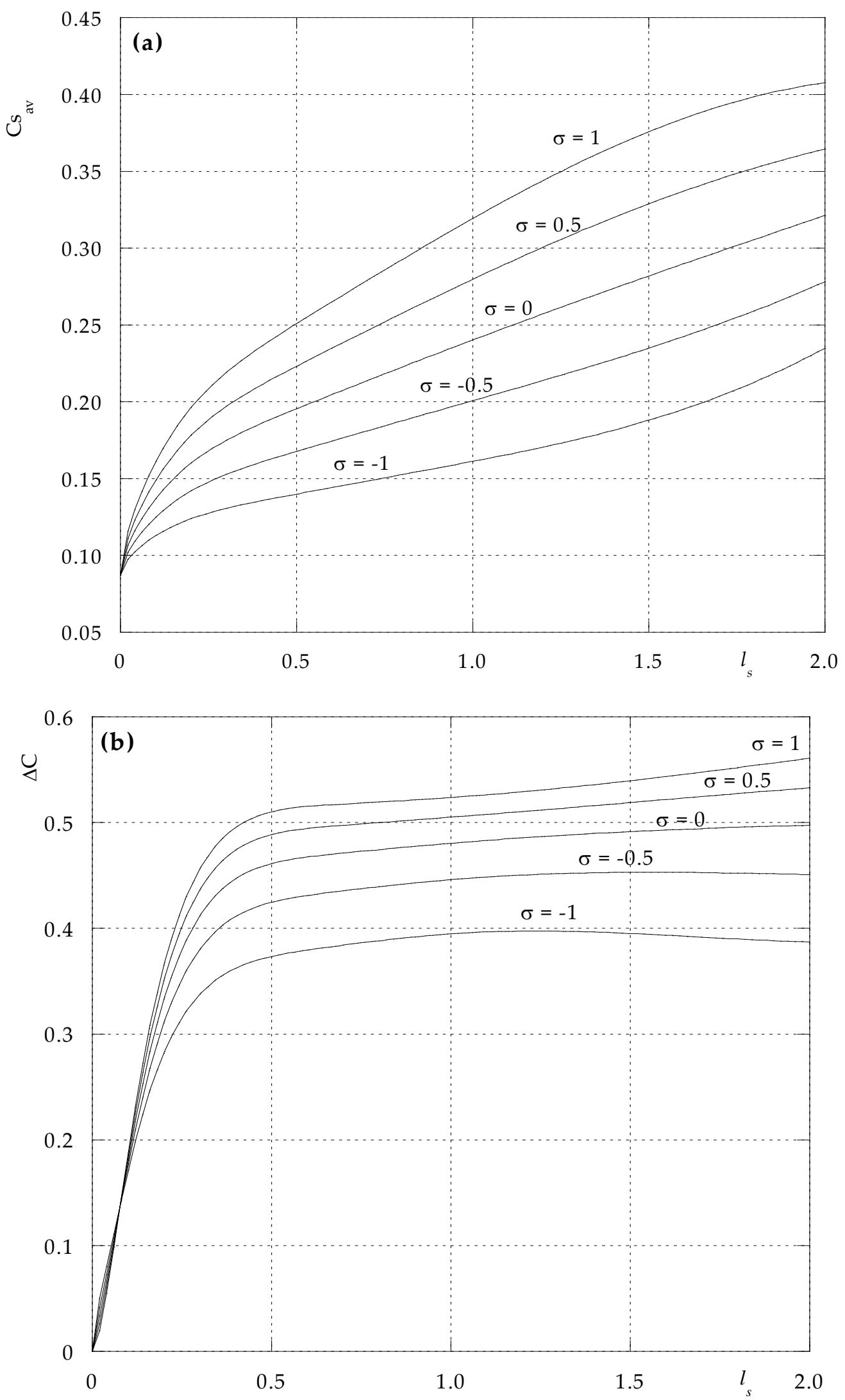

Fig. 4. Variation with $l_{s}$ of the axial (a) and radial (b) segregations during the solidification of half the sample for $\mathrm{Gr}=510^{3}$ and different values of $\sigma$. 

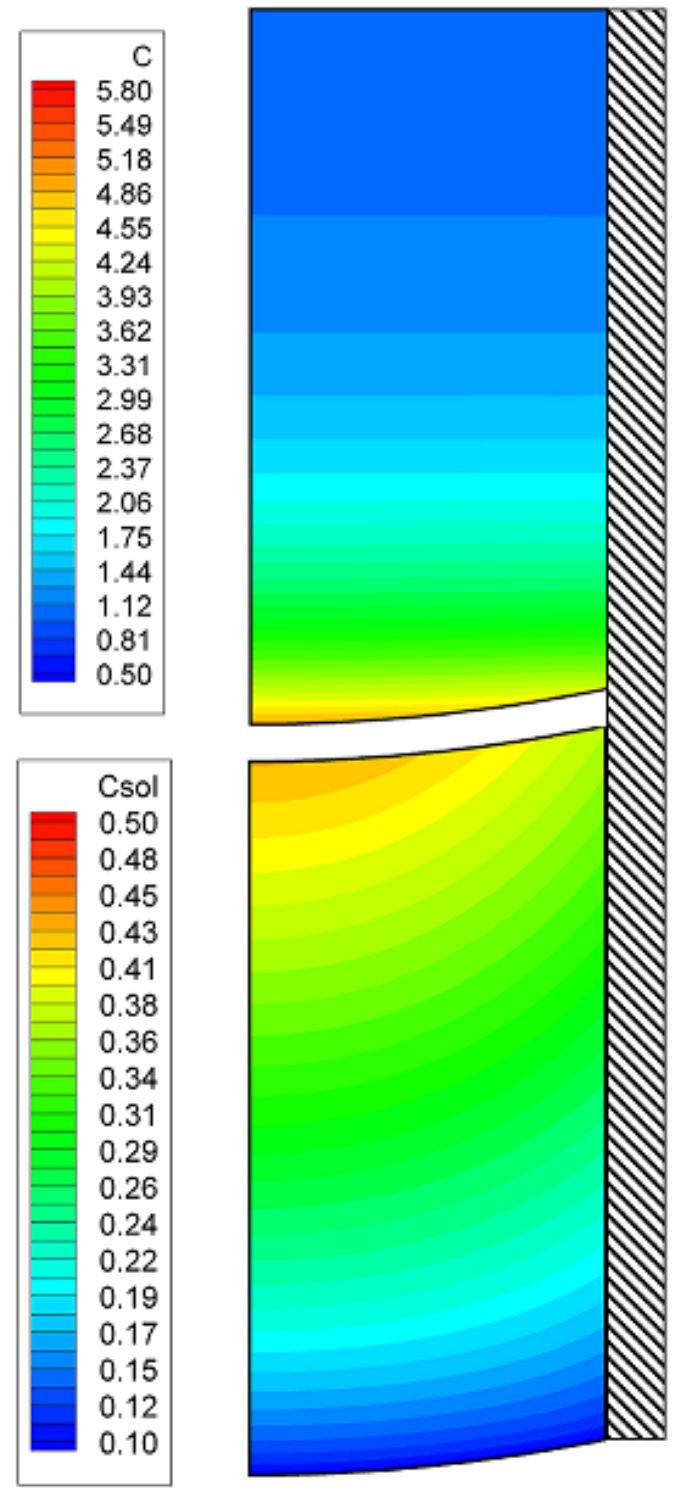

(a)

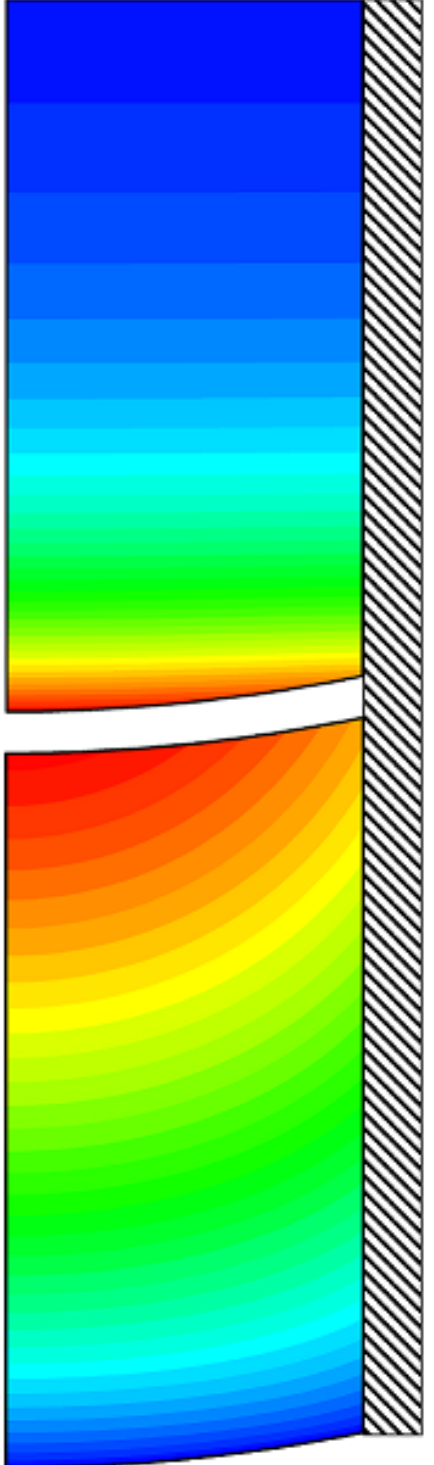

(b)

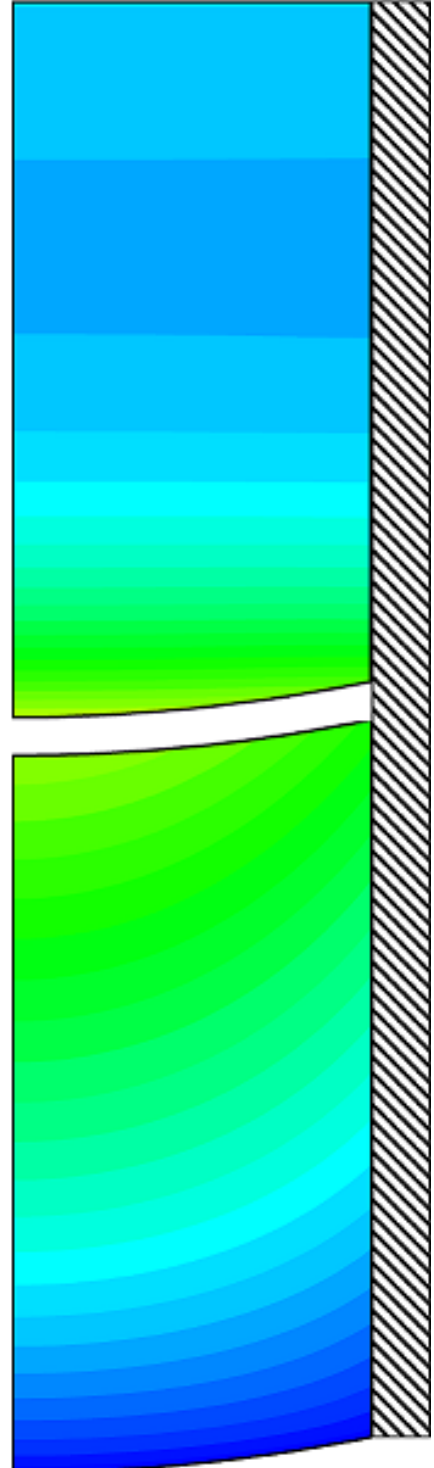

(c)

Fig. 5. Dopant distribution in the melt (top views) and in the crystal (bottom views) when half the sample has been solidified for $\mathrm{Gr}=0, \sigma=0$ (a), $\sigma=0.5$ (b) and $\sigma=-0.5$ (c). The minimum and maximum dopant concentrations in the melt are 1.05 and 4.96 (a), 0.50 and 5.78 (b), 1.40 and 4.10 (c). The maximum dopant concentration in the solid is 0.43 (a), 0.50 (b), 0.35 (c). 


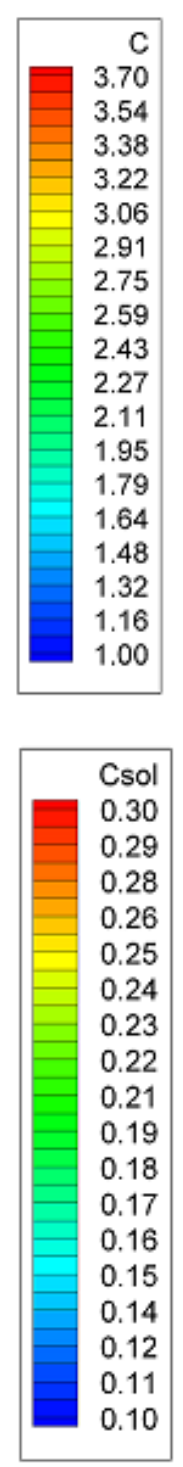

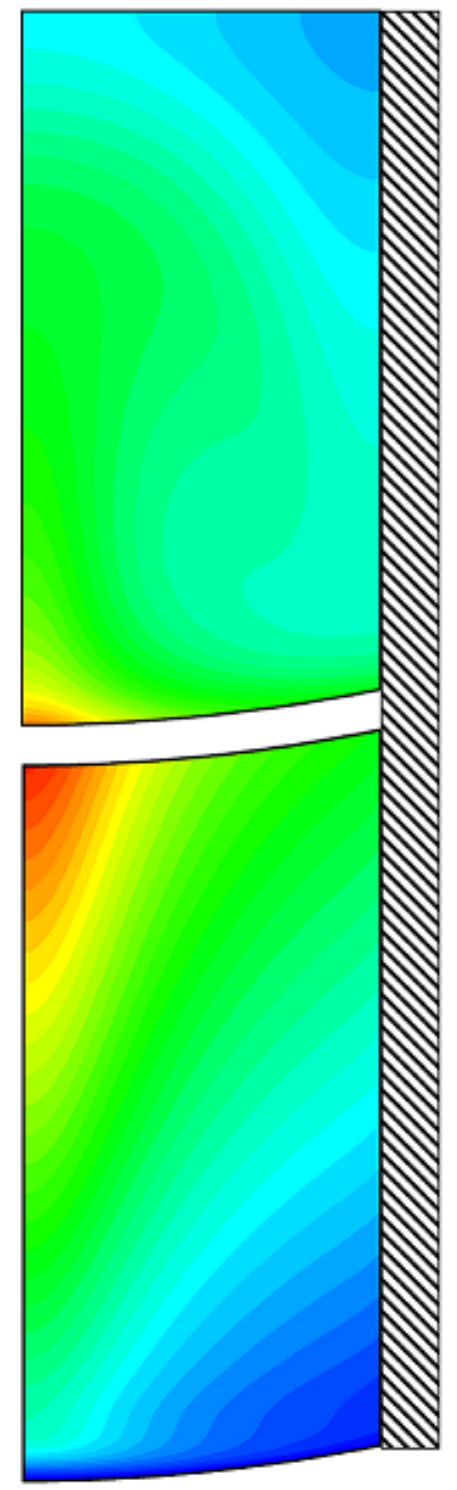

(a)

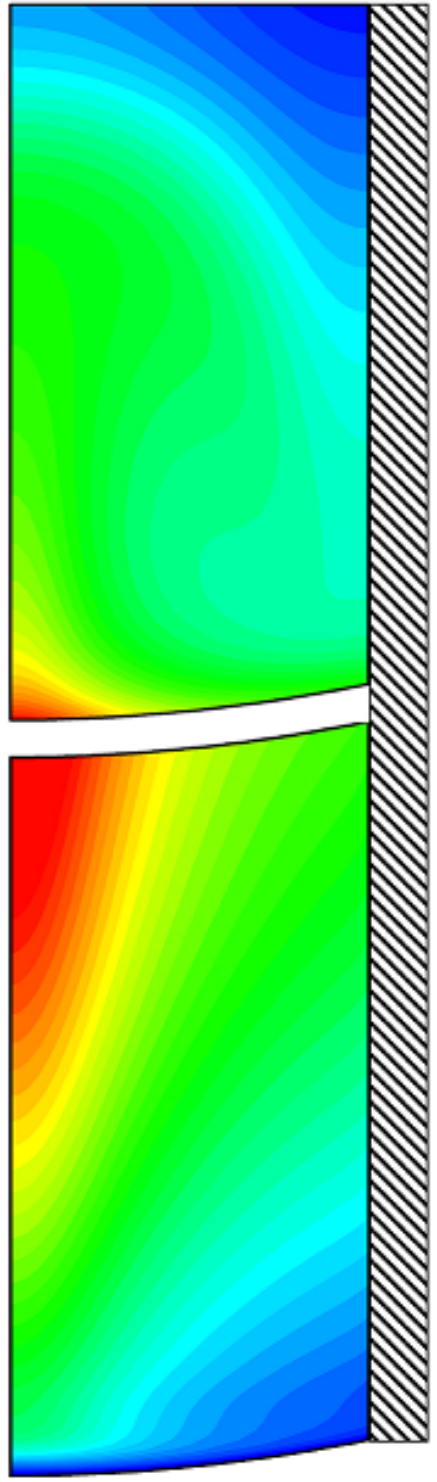

(b)

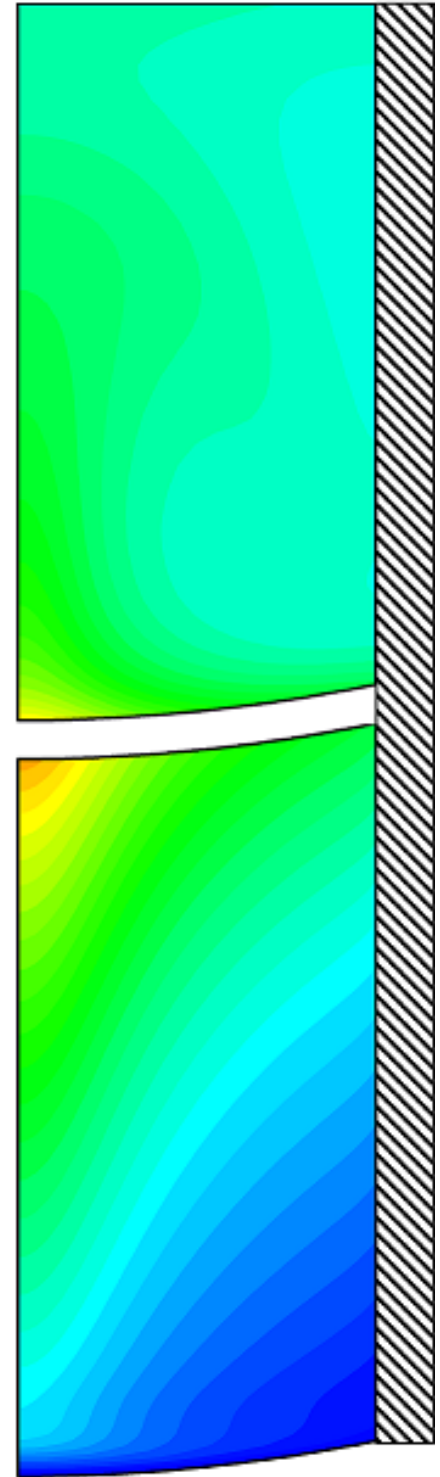

(c)

Fig. 6. Dopant distribution in the melt (top views) and in the crystal (bottom views) when half the sample has been solidified for $\mathrm{Gr}=2.510^{4}, \sigma=0$ (a), $\sigma=0.5$ (b) and $\sigma=-0.5$ (c). The minimum and maximum dopant concentrations in the melt are 1.45 and 3.40 (a), 1.04 and 3.69 (b), 1.75 and 3.09 (c). The maximum dopant concentration in the solid is 0.30 (a), 0.32 (b), 0.27 (c). 

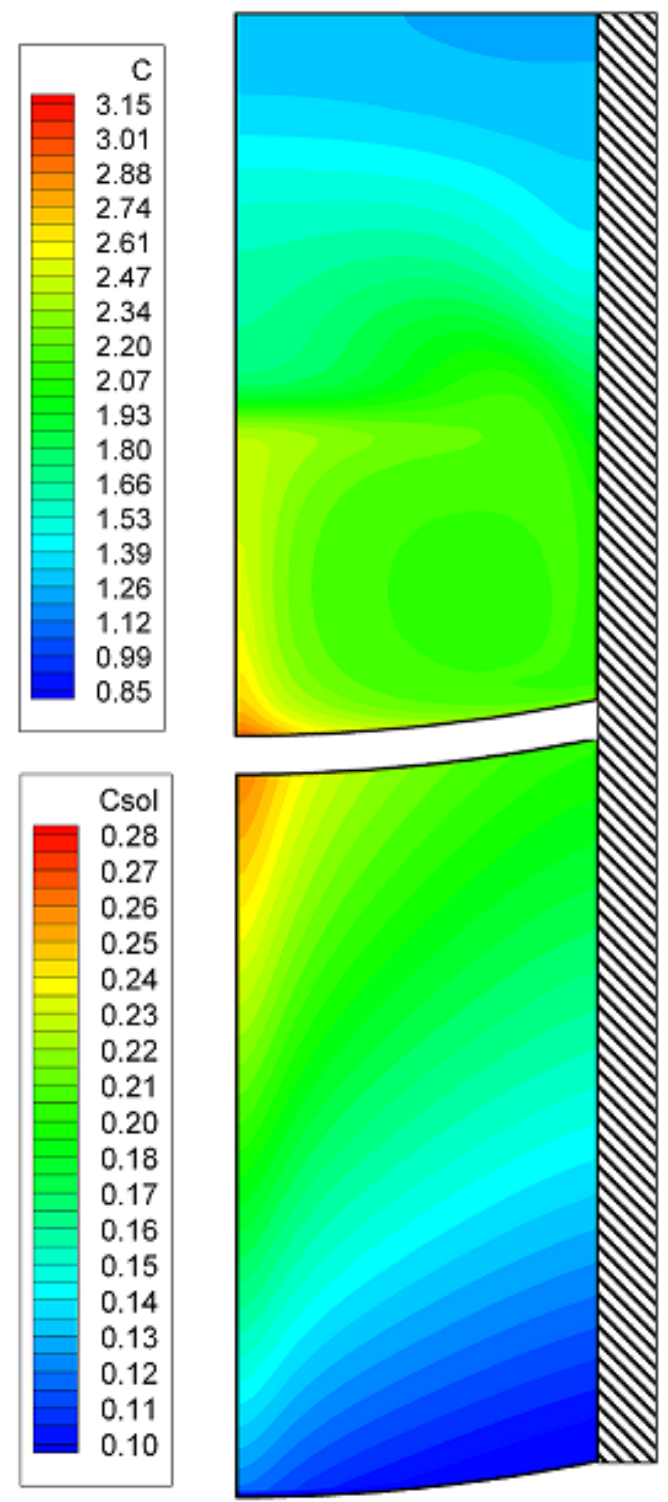

(a)

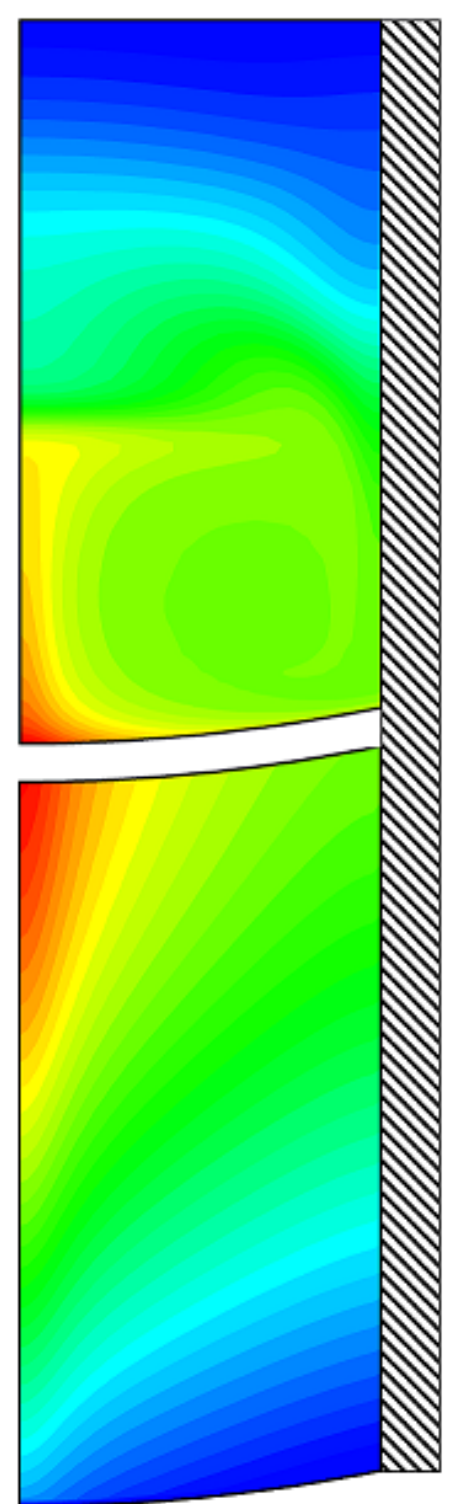

(b)

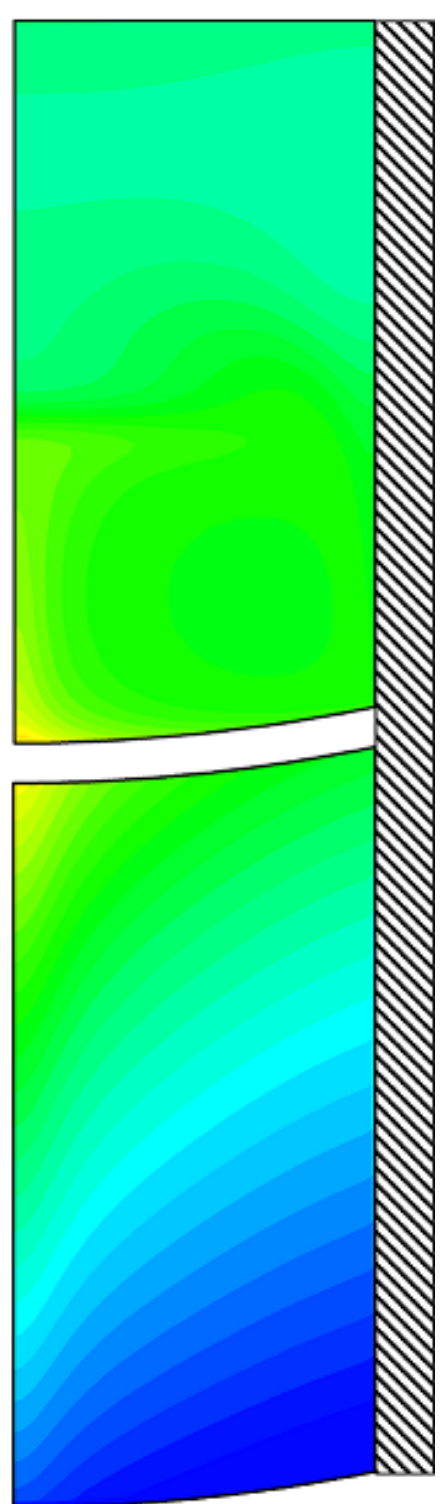

(c)

Fig. 7. Dopant distribution in the melt (top views) and in the crystal (bottom views) when half the sample has been solidified for $\mathrm{Gr}=10^{6}, \sigma=0$ (a), $\sigma=0.5$ (b) and $\sigma=-0.5$ (c). The minimum and maximum dopant concentrations in the melt are 1.24 and 2.97 (a), 0.78 and 3.15 (b), 1.60 and 2.71 (c). The maximum dopant concentration in the solid is 0.26 (a), 0.28 (b), 0.24 (c). 

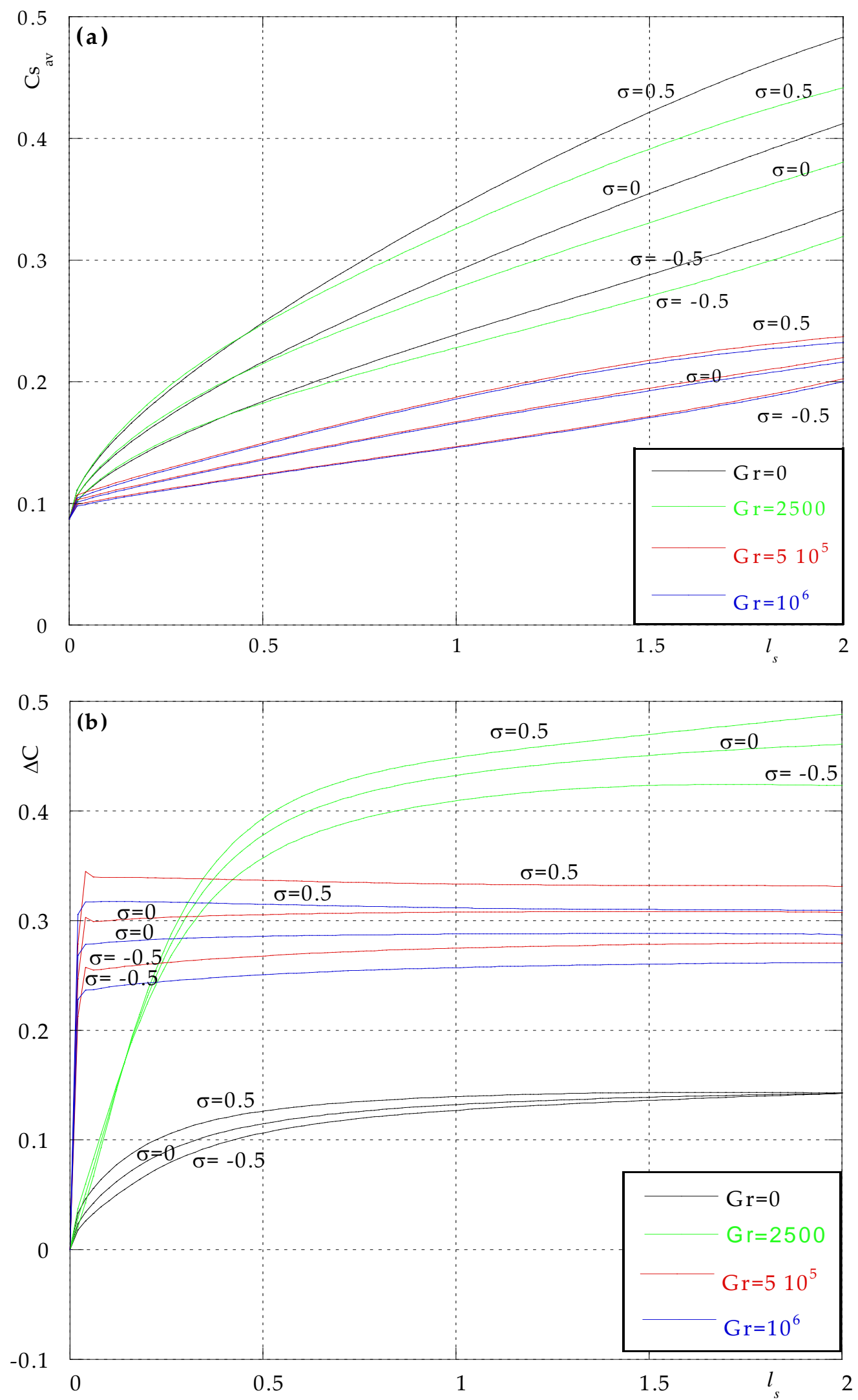

Fig. 8. Variation with $l_{s}$ of the axial (a) and radial (b) segregations during the solidification of half the sample for $\sigma=-0.5,0$ and 0.5 , and different values of $\mathrm{Gr}$. 

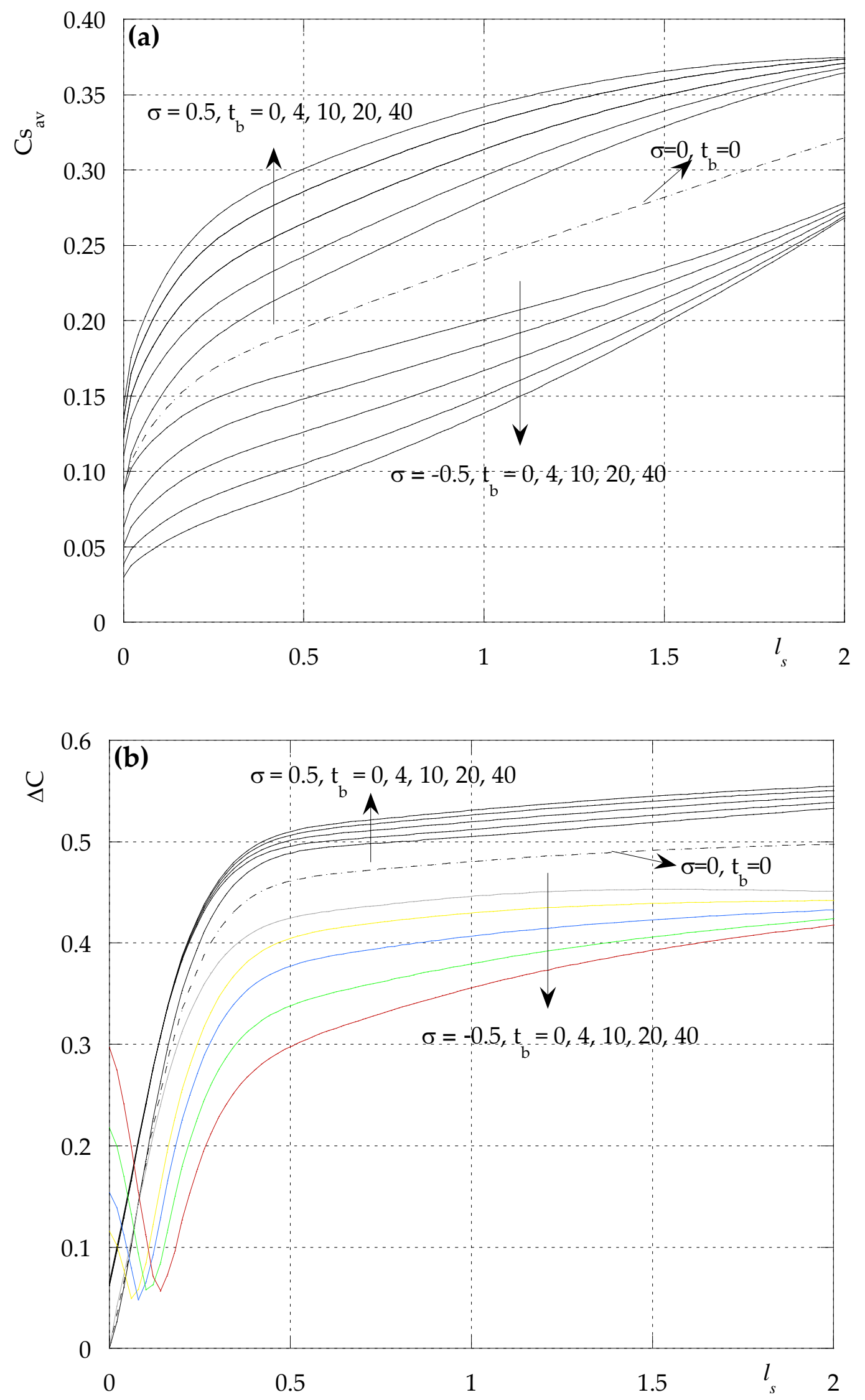

Fig. 9. Variation with $l_{s}$ of the axial (a) and radial (b) segregations during the solidification of half the sample for $\sigma=-0.5,0$ and $0.5, \mathrm{Gr}=510^{3}$ and different values of the furnace residence time $t_{b}$. 